Review Article

\title{
Analytical versus Metaheuristic Methods to Extract the Photovoltaic Cells and Panel Parameters
}

\author{
Daniel T. Cotfas $(\mathbb{D}$, Petru A. Cotfas $(\mathbb{D}$, Mihai P. Oproiu $(\mathbb{D}$, and Paul A. Ostafe \\ Department of Electronics and Computers, Faculty of Electrical Engineering and Computer Science, Transilvania University \\ of Brasov, 500036, Romania \\ Correspondence should be addressed to Daniel T. Cotfas; dtcotfas@unitbv.ro
}

Received 29 May 2021; Revised 11 August 2021; Accepted 24 August 2021; Published 18 September 2021

Academic Editor: Ahmad Umar

Copyright (c) 2021 Daniel T. Cotfas et al. This is an open access article distributed under the Creative Commons Attribution License, which permits unrestricted use, distribution, and reproduction in any medium, provided the original work is properly cited.

\begin{abstract}
The parameters of the photovoltaic cells and panels are very important to forecast the power generated. There are a lot of methods to extract the parameters using analytical, metaheuristic, and hybrid algorithms. The comparison between the widely used analytical method and some of the best metaheuristic algorithms from the algorithm families is made for datasets from the specialized literature, using the following statistical tests: absolute error, root mean square error, and the coefficient of determination. The equivalent circuit and mathematical model considered is the single diode model. The result comparison shows that the metaheuristic algorithms have the best performance in almost all cases, and only for the genetic algorithm, there are poorer results for one chosen photovoltaic cell. The parameters of the photovoltaic cells and panels and also the current-voltage characteristic for real outdoor weather conditions are forecasted using the parameters calculated with the best method: one for analytical-the five-parameter analytical method-and one for the metaheuristic algorithms-hybrid successive discretization algorithm. Additionally, the genetic algorithm is used. The forecast currentvoltage characteristic is compared with the one measured in real sunlight conditions, and the best results are obtained in the case of a hybrid successive discretization algorithm. The maximum power forecast using the calculated parameters with the five-parameter method is the best, and the error in comparison with the measured ones is $0.48 \%$.
\end{abstract}

\section{Introduction}

Nowadays, the power forecasting for the photovoltaic panels and systems plays a very important role for the investors to increase the investments having a realistic scenario. One of the steps to achieve this goal is to accurately and quickly determine the parameters of the photovoltaic cells and panels.

The extraction of the photovoltaic cell parameters is a widely studied issue $[1,2]$, but it remains current due to its importance and the new possibilities created by the metaheuristic algorithms and artificial intelligence [3].

The parameter extraction is possible if there is a dataset which consists of voltage-current pairs $(V, I)$ for the photovoltaic panel, or if the current-voltage characteristic $(I-V)$ is measured. The parameters and dataset can be obtained using the photovoltaic panel datasheet given by the producer [4].
The most commonly used mathematical model to characterize the photovoltaic cells and panels is the single diode model (SD) [1], followed by the double diode model (DD) [5] and rarely three diode model (TD) [6]. The number of the parameters which have to be extracted varies, being five for $\mathrm{SD}$, seven for $\mathrm{DD}$, and nine for TD. There are a lot of methods to extract these parameters, their complexity growing with the increasing number of parameters.

The methods used to extract the parameters of the photovoltaic cells or panels can be classified into analytical, metaheuristic, and hybrid methods [7]. Each of these methods has both advantages and disadvantages.

The contributions and novelty of this paper are as follows:

(i) The main analytical methods and metaheuristic algorithms grouped on families are briefly presented 
(ii) The performance of the methods is analyzed in function of the accuracy with which the parameters are extracted analyzing the absolute error, the root mean square error, and the coefficient of determination

(iii) Choosing the best analytical method considering the following: simplicity of application, the execution time, and the accuracy

(iv) Choosing the metaheuristic algorithm with the smallest root mean square error (RMSE) for different photovoltaic cells and panels from all algorithms considered

(v) Comparing for the first time the analytical method (modified five parameters) and metaheuristic algorithm (hybrid successive discretization algorithm) to forecast the $I-V$ characteristic and the maximum power generated by the commercial monocrystalline photovoltaic panel, giving the manufacturers a tool to choose the best option to characterize the PV for their applications. Additionally, the genetic algorithm is considered in the comparison

The rest of the paper is organized as follows: the equivalent circuits and diode models, statistical tests used for comparison, and the mathematical formulas for calculating the photovoltaic cells and panel parameters at different temperatures and irradiances in the function of their values at the standard test conditions (STC-irradiance $1000 \mathrm{~W} / \mathrm{m}^{2}$, temperature $25^{\circ} \mathrm{C}$, and air mass 1.5) are described in Section 2. A brief presentation of the used methods is made in Section 3. The results and discussions are presented in Section 4, and the last section is dedicated to conclusions and future works.

\section{Methods}

2.1. Photovoltaic Cells and Panel Diode Models. The mathematical model which describes the dependence between the current and the voltage generated by the photovoltaic cells and panels depends on the mechanisms which are taken into account and consequently on the equivalent circuits, Figure 1. The simplest model is the ideal one. The most commonly used model is single diode, Figure 1(a), due to its simplicity but also because it manages to describe the behaviour of most types of photovoltaic cells and panels very well. Equation (1) is the mathematical relation for one diode model:

$$
I=I_{\mathrm{ph}}-I_{\mathrm{o}}\left(e^{\left(V+I R_{\mathrm{s}}\right) / n V_{\mathrm{T}}}-1\right)-\frac{V+I R_{\mathrm{s}}}{R_{\mathrm{sh}}},
$$

where $I_{\mathrm{ph}}$ is the photogenerated current, $I_{\mathrm{o}}$ is the reverse saturation current, $R_{\mathrm{s}}$ is the series resistance, $R_{\mathrm{sh}}$ is the shunt resistance, $n$ is the ideality factor of diode, and $V_{\mathrm{T}}$ is the thermal voltage, $V_{\mathrm{T}}=k T / q . k$ is the Boltzmann constant, $T$ is the temperature, and $q$ is the elementary electrical charge.
The double diode model is described by

$I=I_{\mathrm{ph}}-I_{\mathrm{o} 1}\left(e^{\left(V+I R_{\mathrm{s}}\right) / n_{1} V_{\mathrm{T}}}-1\right)-I_{\mathrm{o} 2}\left(e^{\left(V+I R_{\mathrm{s}}\right) / n_{2} V_{\mathrm{T}}}-1\right)-\frac{V+I R_{\mathrm{s}}}{R_{\mathrm{sh}}}$,

where index 1 relates to the diffusion mechanism and 2 the generation-recombination mechanism. The accuracy to determine the parameters of the photovoltaic cell increases especially at low solar radiation when the two diode model is used [8].

The mathematical model for the photovoltaic panel is described by

$I=N_{\mathrm{p}} I_{\mathrm{ph}}-N_{\mathrm{p}} I_{\mathrm{o}}\left(e^{\left(N_{\mathrm{p}} V+N_{\mathrm{s}} I R_{\mathrm{s}}\right) / n N_{\mathrm{p}} N_{\mathrm{s}} V_{\mathrm{T}}}-1\right)-\frac{N_{\mathrm{p}} V+N_{\mathrm{s}} I R_{\mathrm{s}}}{N_{\mathrm{s}} R_{\mathrm{sh}}}$,

where $N_{\mathrm{s}}$ represents the number of the photovoltaic cells connected in series and $N_{\mathrm{p}}$ represents the number of the photovoltaic cells connected in parallel.

2.2. Statistical Test. The comparison between analytical and metaheuristic algorithms is achieved using different statistical error tests, such as absolute error (AE) Equation (4), the root mean square error Equation (5), and the coefficient of determination $R^{2}$ Equation (6).

$$
\mathrm{AE}=\sum_{i=1}^{n}\left|I_{i \mathrm{c}}-I_{i \mathrm{~m}}\right|
$$

$$
\begin{aligned}
& \text { RMSE }=\sqrt{\frac{\sum_{i=1}^{n}\left(I_{i \mathrm{c}}-I_{i \mathrm{~m}}\right)^{2}}{n}}, \\
& R^{2}=1-\frac{\sum_{i=1}^{n}\left(I_{i \mathrm{c}}-I_{i \mathrm{~m}}\right)^{2}}{\sum_{i=1}^{n}\left(I_{i \mathrm{~m}}-\bar{I}_{i \mathrm{~m}}\right)^{2}},
\end{aligned}
$$

where $I_{i c}$ and $I_{i m}$ represent the calculated and the measured current, respectively, and $n$ is the total number of measurements.

2.3. Irradiance and Temperature Dependence of the $P V$ Parameters. The irradiance and temperature influence more or less the parameters of the photovoltaic cells and panels. The power generated is also dependent on these two factors. So, the relation for photogenerated current, function of the irradiance, and temperature is the following [9]:

$$
I_{\mathrm{ph}}=\frac{G}{G_{\mathrm{ref}}}\left[I_{\mathrm{ph}, \mathrm{ref}}+\alpha_{\mathrm{sc}}\left(T, T_{\mathrm{ref}}\right)\right] \text {, }
$$

where $G$ is irradiance, $T$ represent the temperature, and $\alpha_{\mathrm{sc}}$ is the temperature coefficient of the current. The index ref 


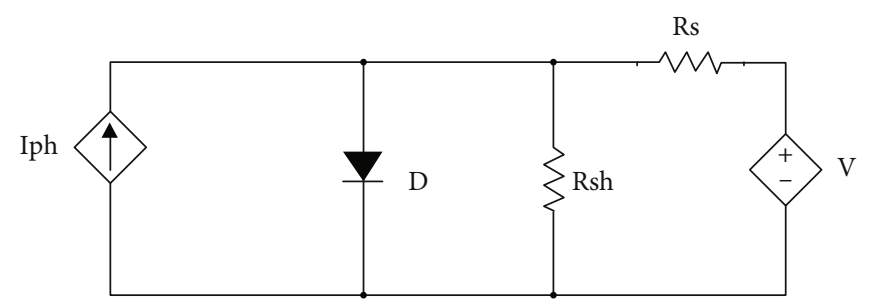

(a)

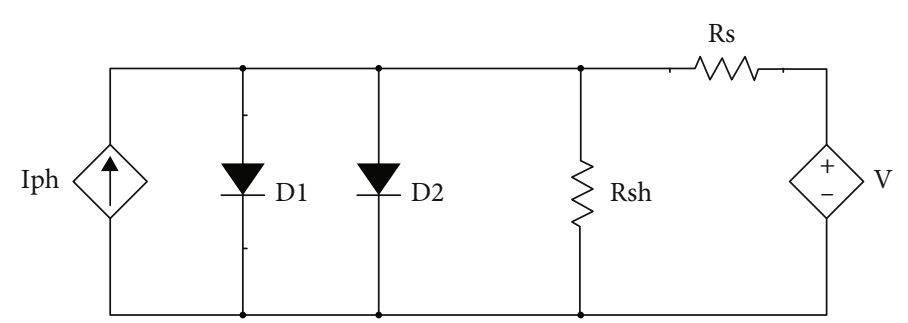

(b)

FIGURE 1: The equivalent circuit of photovoltaic cell: (a) one diode model; (b) two diode model.

is for the parameters at STC. The reverse saturation current can be calculated with Equation (8) [9, 10]:

$$
I_{\mathrm{o}}=I_{\mathrm{o}, \mathrm{ref}}\left(\frac{T}{T_{\text {ref }}}\right)^{3} e^{q / K n\left(\left(E_{g, \text { ref }} / T_{\text {ref }}\right)-\left(E_{g} / T_{r}\right)\right)},
$$

where $E_{g}$ is the energy bandgap. This value depends slightly on temperature $[11,12]$ :

$$
E_{g}=E_{g, \text { ref }}\left\lfloor 1-0.0002677\left(T-T_{\text {ref }}\right)\right\rfloor
$$

The ideality factor of diode depends slightly on the irradiance [13]. The temperature dependence can be written as Equation (10) [9]:

$$
n=n_{\text {ref }} \frac{T}{T_{\text {ref }}} .
$$

The behaviour of the shunt resistance is inversely proportional to that of irradiance, the irradiance increasing as the shunt resistance decreases:

$$
R_{\mathrm{sh}}=R_{\mathrm{sh}, \mathrm{ref}} \frac{G_{\mathrm{ref}}}{G} .
$$

The dependence of the series resistance on temperature and irradiance is described by Equation (12). It decreases linearly with the increase in temperature and increases with the increase of irradiance; $\beta$ is constant and is considered equal to $0.217[11,14]$.

$$
R_{\mathrm{sh}}=R_{\mathrm{sh}, \mathrm{ref}} \frac{T}{T_{\mathrm{ref}}}\left(1-\beta \ln \frac{G}{G_{\mathrm{ref}}}\right) .
$$

\section{Analytical and Metaheuristic Methods}

Pillai and Rajasekar classified the methods to extract the parameters of the photovoltaic cells and panels in analytical, metaheuristic, and hybrid (consisting of those mentioned before) methods [8]. The analytical methods are based on formulas obtained using approximation and/or particular points on the $I-V$ characteristic and some parts of the $I-V$ characteristics. Multiobjective optimization problems were tough issues, but the development and use of metaheuristic algorithms in the last years led to solutions with a very good accuracy [15]. These metaheuristic algorithms were quickly adapted and used to solve the multimodal problem of the current-voltage dependence of the photovoltaic devices.

3.1. Analytical Methods. These methods were used to calculate the parameters of the photovoltaic cells since the $60 \mathrm{~s}$ [13]. A lot of methods have been developed, especially for the SD model, but in the last years, they were developed for DD and even TD models. They can calculate one, more than one, or all parameters of the photovoltaic cells and panels. The several analytical methods are presented in Table 1.

The complexity of usage and the accuracy of the method to extract the parameters of the photovoltaic cells or panels are two key indicators. Three-level ratings are used for each of them: low, medium, and high. For the complexity of usage, they mean as follows: low: simple formulas are used; medium: complex formulas, fitting and iterative procedure are necessary; high: the analytical method needs dedicated computational software [29]. For the accuracy, the level rating is the function of the statistical test $[29,30]$. The rating for each method is shown in Table 1 . Their results can be used by the manufacturers to choose the optimum method to characterize the photovoltaic cells.

The analytical five-parameter method, $5 \mathrm{P}$, is the most widely used of the analytical ones to extract the parameters of the photovoltaic cells. The first step is to calculate the 
TABLe 1: The analytical methods.

\begin{tabular}{|c|c|c|c|c|c|c|}
\hline Methods & Parameters & Models & Remarks & $\begin{array}{l}\text { Complexity } \\
\text { of usage }\end{array}$ & Accuracy & Ref. \\
\hline $\begin{array}{l}\text { Analytical five- } \\
\text { parameter } \\
\text { method }\end{array}$ & $I_{\mathrm{ph}}, I_{\mathrm{o}}, n, R_{\mathrm{s}}, R_{\mathrm{sh}}$ & SD & $\begin{array}{l}\text { Using part of } I-V \text { characteristic to } \\
\text { determine the } R_{\mathrm{s}} \text { and } R_{\mathrm{sh}}\end{array}$ & Medium & High & {$[16]$} \\
\hline Tivanov & $I_{\mathrm{ph}}, I_{\mathrm{o}}, n, R_{\mathrm{s}}, R_{\mathrm{sh}}$ & SD & $\begin{array}{l}\text { Using part of } I-V \text { characteristic to determine } \\
\text { the } R_{\mathrm{s}} \text { and } R_{\mathrm{sh}} \text { and } I_{\mathrm{ph}} \sim I_{\mathrm{sc}}\end{array}$ & Medium & Medium & {$[17]$} \\
\hline Ortiz-Conde & $I_{\mathrm{ph}}, I_{\mathrm{o}}, n, R_{\mathrm{s}}, \mathrm{R}_{\mathrm{sh}}$ & SD & $\begin{array}{l}\text { Using the CC function to calculate the equation } \\
\text { coefficients } C_{\mathrm{V} 1}, C_{\mathrm{I} 1}, C_{\mathrm{V} 2} \text { and } C_{\mathrm{I} 2}\end{array}$ & Low & Medium & {$[18]$} \\
\hline Garrido-Alzar & $I_{\mathrm{ph}}, I_{\mathrm{od}}, I_{\mathrm{or}}, n_{\mathrm{r}}, R_{\mathrm{s}}, R_{\mathrm{sh}}$ & DD & $n_{\mathrm{r}}$ is considered 1 , and four points $(V, I)$ are using & Medium & Low & {$[19]$} \\
\hline Generalized area & $n, R_{\mathrm{s}}, R_{\mathrm{sh}}$ & $\mathrm{SD}$ & $\begin{array}{c}\text { Using } I_{\mathrm{ph}} \sim I_{\mathrm{sc}} \text {, and the subgraphic area for three } I-V \\
\text { characteristics }\end{array}$ & High & Low & {$[20]$} \\
\hline Area & $R_{\mathrm{s}}$ & SD & Using subgraphic area for $I-V$ characteristic and $n=1$ & Low & Low & {$[21]$} \\
\hline Kaminski & $I_{\mathrm{od}}, I_{\mathrm{or}}, n_{\mathrm{r}}, R_{\mathrm{s}}, R_{\mathrm{sh}}$ & DD & The parameters are determined in dark conditions & Low & Low & {$[22]$} \\
\hline$R_{\mathrm{s}}$ model & $I_{\mathrm{od}}, I_{\mathrm{or}}, n_{\mathrm{r}}, R_{\mathrm{s}}$ & SD & $R_{\mathrm{sh}}$ is considered $\infty$ & Medium & Low & {$[23]$} \\
\hline L function & $R_{\mathrm{s}}, R_{\mathrm{sh}}$ & $\mathrm{SD}$ & Using Lambert $\mathrm{W}$ function & High & Medium & {$[24]$} \\
\hline $\begin{array}{l}\text { Explicit method } \\
\text { for the five } \\
\text { parameters }\end{array}$ & $I_{\mathrm{ph}}, I_{\mathrm{o}}, n, R_{\mathrm{s}}, R_{\mathrm{sh}}$ & SD & $\begin{array}{l}\text { Using two points for } I_{\mathrm{sc}} \text { zone and } V_{\mathrm{oc}} \\
\text { zone to calculate the slopes }\end{array}$ & Medium & Medium & [9] \\
\hline Cotfas & $R_{\mathrm{s}}$ and $s$ & $\mathrm{SD}$ & Using $I-V$ characteristic measured and the ideal one & Medium & Medium & {$[1]$} \\
\hline $\begin{array}{l}\text { Modified five } \\
\text { parameters }\end{array}$ & $I_{\mathrm{ph}}, I_{\mathrm{o}}, n, R_{\mathrm{s}}, R_{\mathrm{sh}}$ & $\mathrm{SD}$ & Using two empirically equations to calculate $R_{\mathrm{s}}$ and $R_{\mathrm{sh}}$ & Low & High & {$[25]$} \\
\hline Elkholy & $I_{\mathrm{ph}}, I_{\mathrm{o}}, n, R_{\mathrm{s}}, R_{\mathrm{sh}}$ & SD & $\begin{array}{l}\text { Using a method based on the nonlinear least-squares } \\
\text { algorithm; the parameters are calculated at different } \\
\text { environmental conditions }\end{array}$ & Medium & Medium & [7] \\
\hline Ndegwa & $I_{\mathrm{ph}}, I_{\mathrm{o}}, n, R_{\mathrm{s}}, R_{\mathrm{sh}}$ & $\mathrm{SD}$ & $\begin{array}{l}n \text { and } I_{\mathrm{o}} \text { are calculated firstly using } I_{\mathrm{sc}}, I_{\mathrm{m}}, V_{\mathrm{m}}, V_{\mathrm{oc}} \text {, and } \\
R_{\mathrm{s}}=0, R_{\mathrm{sh}} \approx \infty ; R_{\mathrm{s}} \text { and } R_{\mathrm{sh}} \text { are then evaluated for } \\
\text { different values of } n_{\mathrm{s}} \text { in the neighborhood of } n_{\mathrm{o}} \\
\left(1 \leq n \leq n_{\mathrm{o}}\right)\end{array}$ & Medium & Medium & {$[26]$} \\
\hline TRDLA & $I_{\mathrm{ph}}, I_{\mathrm{o}}, n, R_{\mathrm{s}}, R_{\mathrm{sh}}$ & SD & $\begin{array}{l}n \text { is calculated firstly using the data provided by the } \\
\text { manufacturer's datasheet; the other four parameters are } \\
\text { calculated using the trust-region-dogleg algorithm }\end{array}$ & Medium & Medium & {$[27]$} \\
\hline Brano & $I_{\mathrm{ph}}, I_{\mathrm{o}}, n, R_{\mathrm{s}}, R_{\mathrm{sh}}$ & $\mathrm{SD}$ & Using five equations derived from Equation (1) & Medium & Medium & {$[28]$} \\
\hline
\end{tabular}

slope around the open-circuit voltage to calculate $R_{\mathrm{so}}$, Equation (12), and the short circuit current to calculate $R_{\text {sho' }}$, Equation (13). The slope calculation requires having many points on the $I-V$ characteristic in the two regions. In case this is not possible and to reduce the complexity of the method, $R_{\text {so }}$ and $R_{\text {sho }}$ can be calculated using Equations (15) and (16), empirically obtained in modified five parameters 5Pm [25].

$$
\begin{gathered}
R_{\mathrm{so}}=-\left(\frac{d V}{d I}\right)_{V=V_{\mathrm{oc}}}, \\
R_{\mathrm{sh}}=R_{\mathrm{sho}}=-\left(\frac{d V}{d I}\right)_{I=I_{\mathrm{sc}}} \\
R_{\mathrm{so}}=0.002102+0.318070 R_{\mathrm{sm}}, R_{\mathrm{sm}}=\frac{V_{\mathrm{oc}}-V_{\mathrm{m}}}{I_{\mathrm{m}}},
\end{gathered}
$$

$$
R_{\text {sho }}=-0.051914+2.505219 R_{\mathrm{sm}}, R_{\mathrm{shm}}=\frac{V_{\mathrm{m}}}{I_{\mathrm{sc}}-I_{\mathrm{m}}},
$$

where $I_{\mathrm{m}}$ and $V_{\mathrm{m}}$ represent the maximum power point coordinates.

$$
\begin{gathered}
n=\frac{V_{\mathrm{m}}+R_{\mathrm{so}} I_{\mathrm{m}}-V_{\mathrm{oc}}}{V_{t}\left(\ln \left(I_{\mathrm{sc}}-\left(V_{\mathrm{m}} / R_{\mathrm{sh}}\right)-I_{\mathrm{m}}\right)-\ln \left(I_{\mathrm{sc}}-\left(V_{\mathrm{oc}} / R_{\mathrm{sh}}\right)\right)+I_{\mathrm{m}} /\left(I_{\mathrm{sc}}-\left(V_{\mathrm{oc}} / R_{\mathrm{sh}}\right)\right)\right)}, \\
I_{\mathrm{o}}=\left(I_{\mathrm{sc}}-\frac{V_{\mathrm{oc}}}{R_{\mathrm{sh}}}\right) \exp \left(-\frac{V_{\mathrm{oc}}}{n V_{\mathrm{T}}}\right), \\
R_{\mathrm{s}}=R_{\mathrm{so}}-\frac{n V_{\mathrm{t}}}{I_{\mathrm{o}}} \exp \left(-\frac{V_{\mathrm{oc}}}{n V_{\mathrm{T}}}\right), \\
I_{\mathrm{ph}}=I_{\mathrm{sc}}\left(1+\frac{R_{\mathrm{s}}}{R_{\mathrm{sh}}}\right)+I_{\mathrm{o}}\left(\exp \left(\frac{I_{\mathrm{sc}} R_{\mathrm{s}}}{n V_{\mathrm{T}}}\right)-1\right) .
\end{gathered}
$$

3.2. Metaheuristic Methods. The metaheuristic algorithms have been used to extract the parameters of the PV since the 2000s, when Jervase et al. used the genetic algorithms 
TABle 2: The metaheuristic algorithms.

\begin{tabular}{|c|c|c|c|c|c|c|}
\hline Family algorithms & Type & Models & PV & Range set & $\begin{array}{l}\text { Computational } \\
\text { time/iterations }\end{array}$ & Reference \\
\hline Genetic & Simple & SD & $50 \mathrm{~W}$ panel & Partially & $-/ 50$ & [31] \\
\hline Genetic & Simple & SD & $57 \mathrm{~mm}$ RTC France solar cell & Yes & $-/-$ & {$[32]$} \\
\hline GA-R & Simple & SD & $\begin{array}{l}57 \text { mm RTC France solar cell, mSi } \\
\text { commercial photovoltaic cell }\end{array}$ & Partially & $56 s / 5000$ & {$[33]$} \\
\hline Genetic GA-LS & Hybrid & $\mathrm{SD}$ & $57 \mathrm{~mm}$ RTC France solar cell & Yes & $-/-$ & {$[34]$} \\
\hline Differential evolution DE & Simple & $\begin{array}{l}\mathrm{SD} \\
\mathrm{DD}\end{array}$ & $\begin{array}{l}57 \mathrm{~mm} \text { RTC France solar cell, PWP201 } \\
\text { photovoltaic panel }\end{array}$ & Yes & $\begin{array}{l}12 \mathrm{~s} \text { and } 16 \mathrm{~s} / \\
10000 \text { and } \\
20000\end{array}$ & [35] \\
\hline $\begin{array}{l}\text { Differential evolution } \\
\mathrm{R}_{\mathrm{cr}} \text {-IJADE }\end{array}$ & Simple & $\begin{array}{l}\mathrm{SD} \\
\mathrm{DD}\end{array}$ & $57 \mathrm{~mm}$ RTC France solar cell & Yes & $\begin{array}{l}33 \mathrm{~s} \text { and } 58 \mathrm{~s} / \\
10000 \text { and } \\
20000\end{array}$ & {$[36]$} \\
\hline $\begin{array}{l}\text { Penalty differential } \\
\text { evolution P-DE }\end{array}$ & Simple & $\mathrm{DD}$ & $\begin{array}{l}\text { pSi-S75 and S115 mSi-SM55 and SQ150PC } \\
\text { tin film-ST36 and ST40 }\end{array}$ & Partially & $42 \mathrm{~s} / 500$ & {$[37]$} \\
\hline $\begin{array}{l}\text { Differential evolution } \\
\text { with an individual- } \\
\text { dependent mechanism } \\
\text { IDE }\end{array}$ & Simple & $\begin{array}{l}\mathrm{SD} \\
\mathrm{DD}\end{array}$ & $\begin{array}{l}57 \mathrm{~mm} \text { RTC France solar cell, PWP201 } \\
\text { photovoltaic panel }\end{array}$ & Yes & $\begin{array}{l}-/ 10000 \text { and } \\
20000\end{array}$ & {$[35]$} \\
\hline $\begin{array}{l}\text { Linear population } \\
\text { success-history-based } \\
\text { adaptive DE L-SHADE }\end{array}$ & Simple & $\begin{array}{l}\mathrm{SD} \\
\mathrm{DD}\end{array}$ & $\begin{array}{l}57 \mathrm{~mm} \text { RTC France solar cell, PWP201 } \\
\text { photovoltaic panel }\end{array}$ & Yes & $\begin{array}{l}35.4 \text { and } \\
62.66 \mathrm{~s} / 10000 \\
\text { and } 20000\end{array}$ & {$[35]$} \\
\hline $\begin{array}{l}\text { Differential evolution } \\
\text { DEIM }\end{array}$ & Hybrid & $\begin{array}{l}\mathrm{SD} \\
\mathrm{DD}\end{array}$ & KC120 PV module & Yes & $\begin{array}{l}32 \mathrm{~s} / 10000 \text { and } \\
20000\end{array}$ & {$[38]$} \\
\hline $\begin{array}{l}\text { Particle swarm } \\
\text { optimization CPSO }\end{array}$ & Simple & SD & $57 \mathrm{~mm}$ RTC France solar cell & Yes & $-/ 4500$ & {$[39]$} \\
\hline $\begin{array}{l}\text { Particle swarm } \\
\text { optimization VCPSO }\end{array}$ & Simple & $\mathrm{DD}$ & - & No & $-/-$ & {$[40]$} \\
\hline $\begin{array}{l}\text { Particle swarm } \\
\text { optimization NM-MPSO }\end{array}$ & Hybrid & $\begin{array}{l}\mathrm{SD} \\
\mathrm{DD}\end{array}$ & $57 \mathrm{~mm}$ RTC France solar cell & Yes & $-/ 350000$ & {$[41]$} \\
\hline $\begin{array}{l}\text { Fractional chaotic } \\
\text { ensemble particle swarm } \\
\text { optimizer FC-EPSO }\end{array}$ & Hybrid & $\begin{array}{l}\mathrm{SD} \\
\mathrm{DD}\end{array}$ & $57 \mathrm{~mm}$ RTC France solar cell & Yes & $\begin{array}{l}11.5 \mathrm{~s} \text { and } 12 \mathrm{~s} / \\
200\end{array}$ & {$[42]$} \\
\hline $\begin{array}{l}\text { Chaotic heterogeneous } \\
\text { comprehensive learning } \\
\text { PSO C-HCLPSO }\end{array}$ & Hybrid & $\begin{array}{l}\mathrm{SD} \\
\mathrm{DD}\end{array}$ & 57 mm RTC France solar cell & Yes & $\begin{array}{l}204 \mathrm{~s} \text { and } 225 \mathrm{~s} / \\
2000\end{array}$ & {$[43]$} \\
\hline $\begin{array}{l}\text { Hybrid successive } \\
\text { discretization algorithm } \\
\text { HSDA }\end{array}$ & Hybrid & $\begin{array}{l}\mathrm{SD} \\
\mathrm{DD}\end{array}$ & $\begin{array}{l}57 \mathrm{~mm} \text { RTC France solar cell, } 3 \times 3 \mathrm{~cm} \\
\text { monocrystalline silicon photovoltaic cell, PWP201 } \\
\text { photovoltaic panel, STP6-120/36, STM6-40/36, etc. }\end{array}$ & Yes & $28 s$ and $46 s / 4$ & {$[44]$} \\
\hline Discretization SDA & Simple & $\mathrm{SD}$ & $\begin{array}{l}57 \mathrm{~mm} \text { RTC France solar cell, } 3 \times 3 \mathrm{~cm} \\
\text { monocrystalline silicon photovoltaic cell, PWP201 } \\
\text { photovoltaic panel }\end{array}$ & Yes & $\begin{array}{l}142 \mathrm{~s} \text { and } 266 \mathrm{~s} / \\
4\end{array}$ & {$[3]$} \\
\hline Discretization PSDA & Simple & $\begin{array}{l}\mathrm{SD} \\
\mathrm{DD}\end{array}$ & $\begin{array}{l}57 \text { mm RTC France solar cell, PWP201 photovoltaic } \\
\text { panel, Kyocera KC200GT photovoltaic panel }\end{array}$ & Yes & $28 s$ and $46 s / 4$ & {$[45]$} \\
\hline $\begin{array}{l}\text { Artificial bee colony } \\
\text { optimization ABCO }\end{array}$ & Simple & $\begin{array}{l}\mathrm{SD} \\
\mathrm{DD}\end{array}$ & $\begin{array}{l}57 \mathrm{~mm} \text { RTC France solar cell, PWP201 photovoltaic } \\
\text { panel, STM6-40/36 }\end{array}$ & Yes & $-/ 10000$ & {$[46]$} \\
\hline $\begin{array}{l}\text { Artificial bee colony } \\
\text { optimization ABC-NMS }\end{array}$ & Hybrid & SD & $\begin{array}{l}57 \mathrm{~mm} \text { RTC France solar cell, PWP201 } \\
\text { photovoltaic panel }\end{array}$ & Yes & $-/ 5000$ & {$[47]$} \\
\hline $\begin{array}{l}\text { Shuffled complex } \\
\text { evolution ISCE }\end{array}$ & Simple & $\begin{array}{l}\mathrm{SD} \\
\mathrm{DD}\end{array}$ & $\begin{array}{l}57 \mathrm{~mm} \text { RTC France solar cell, PWP201 } \\
\text { photovoltaic panel }\end{array}$ & Yes & $\begin{array}{l}-/ 5000 \text { and } \\
10000\end{array}$ & {$[48]$} \\
\hline $\begin{array}{l}\text { Shuffled complex } \\
\text { evolution-opposition- } \\
\text { based learning ESCE- } \\
\text { OBL }\end{array}$ & Hybrid & $\begin{array}{l}\mathrm{SD} \\
\mathrm{DD}\end{array}$ & $\begin{array}{l}57 \mathrm{~mm} \text { RTC France solar cell, PWP201 } \\
\text { photovoltaic panel }\end{array}$ & - & $\begin{array}{l}-/ 5000 \text { and } \\
10000\end{array}$ & [49] \\
\hline Simulated annealing SA & Simple & $\begin{array}{l}\mathrm{SD} \\
\mathrm{DD}\end{array}$ & 57 mm R.T.C France solar cell, Photowatt-PWP201 & - & $-1-$ & {$[64]$} \\
\hline
\end{tabular}


TABLE 2: Continued.

\begin{tabular}{|c|c|c|c|c|c|c|}
\hline Family algorithms & Type & Models & PV & Range set & $\begin{array}{l}\text { Computational } \\
\text { time/iterations }\end{array}$ & Reference \\
\hline $\begin{array}{l}\text { Simulated annealing LM- } \\
\text { SA }\end{array}$ & Hybrid & SD & 57 mm RTC France solar cell & - & $-/ 2050$ & {$[50]$} \\
\hline Flower pollination FPA & Simple & $\begin{array}{l}\mathrm{SD} \\
\mathrm{DD}\end{array}$ & 57 mm RTC France solar cell, Photowatt-PWP201 & Yes & $-/ 25000$ & {$[51]$} \\
\hline $\begin{array}{l}\text { Flower pollination } \\
\text { BPFPA }\end{array}$ & Hybrid & $\begin{array}{l}\mathrm{SD} \\
\mathrm{DD}\end{array}$ & 57 mm RTC France solar cell & Partially & $-/ 20000$ & {$[52]$} \\
\hline Harmony search HS & Simple & $\begin{array}{l}\mathrm{SD} \\
\mathrm{DD}\end{array}$ & 57 mm RTC France solar cell & - & $-/ 5000$ & {$[53]$} \\
\hline $\begin{array}{l}\text { Innovative global } \\
\text { harmony search IGHS }\end{array}$ & Simple & $\begin{array}{l}\mathrm{SD} \\
\mathrm{DD}\end{array}$ & 57 mm RTC France solar cell & - & $-/ 5000$ & {$[53]$} \\
\hline Pattern search PS & Simple & $\begin{array}{l}\mathrm{SD} \\
\mathrm{DD}\end{array}$ & $\begin{array}{l}57 \mathrm{~mm} \text { RTC France solar cell, PWP201 } \\
\text { photovoltaic panel }\end{array}$ & - & $-1-$ & {$[54]$} \\
\hline JAYA algorithm IJAYA & Simple & $\begin{array}{l}\mathrm{SD} \\
\mathrm{DD}\end{array}$ & $\begin{array}{l}57 \mathrm{~mm} \text { RTC France solar cell, PWP201 } \\
\text { photovoltaic panel }\end{array}$ & Yes & $-/ 50000$ & {$[55]$} \\
\hline $\begin{array}{l}\text { Performance-guided } \\
\text { JAYA algorithm PGJAYA }\end{array}$ & Simple & $\begin{array}{l}\mathrm{SD} \\
\mathrm{DD}\end{array}$ & $\begin{array}{l}57 \text { mm R.T.C France solar cell, Photowatt-PWP201 } \\
\text { KC200GT, SM55, thin-film Shell ST40 }\end{array}$ & Yes & $-/ 50000$ & {$[56]$} \\
\hline $\begin{array}{l}\text { Comprehensive learning } \\
\text { JAYA algorithm CJAYA }\end{array}$ & Simple & $\begin{array}{l}\mathrm{SD} \\
\mathrm{DD}\end{array}$ & $\begin{array}{l}57 \mathrm{~mm} \text { RTC France solar cell, PWP201 } \\
\text { photovoltaic panel }\end{array}$ & - & $\begin{array}{l}-/ 20000 \text { and } \\
48000\end{array}$ & {$[57]$} \\
\hline $\begin{array}{l}\text { Teaching-learning-based } \\
\text { optimization TLBO }\end{array}$ & Simple & $\begin{array}{l}\mathrm{SD} \\
\mathrm{DD}\end{array}$ & $\begin{array}{l}57 \mathrm{~mm} \text { RTC France solar cell, PWP201 } \\
\text { photovoltaic panel }\end{array}$ & - & $-/ 20000$ & {$[58]$} \\
\hline Improved TLBO ITLBO & Simple & $\begin{array}{l}\mathrm{SD} \\
\mathrm{DD}\end{array}$ & $\begin{array}{c}57 \mathrm{~mm} \text { R.T.C France solar cell, PWP201 } \\
\text { photovoltaic panel, STP6-120/36, STM6-40/36 }\end{array}$ & - & $\begin{array}{c}5.95 \mathrm{~s}(30) \text { and } \\
6.60 \mathrm{~s}(30) / \\
50000\end{array}$ & {$[59]$} \\
\hline $\begin{array}{l}\text { Whale optimization } \\
\text { algorithm WOA }\end{array}$ & Simple & $\begin{array}{l}\mathrm{SD} \\
\mathrm{DD}\end{array}$ & KC200GT & Yes & $-/ 45000$ & {$[60]$} \\
\hline $\begin{array}{l}\text { Improved version of } \\
\text { WOA IWOA }\end{array}$ & Simple & $\begin{array}{l}\mathrm{SD} \\
\mathrm{DD}\end{array}$ & $\begin{array}{c}57 \text { mm RTC France solar cell, PWP201 photovoltaic } \\
\text { panel, JAM6-60-295W-4BB, CS6U-320P }\end{array}$ & Yes & $-/ 100000$ & {$[61]$} \\
\hline $\begin{array}{l}\text { Multiple learning } \\
\text { backtracking search } \\
\text { algorithm MLBSA }\end{array}$ & Simple & $\begin{array}{l}\mathrm{SD} \\
\mathrm{DD}\end{array}$ & $\begin{array}{l}57 \mathrm{~mm} \text { RTC France solar cell, PWP201 } \\
\text { photovoltaic panel }\end{array}$ & Yes & $\begin{array}{l}39 \text { and } 44 \mathrm{~s} / \\
50000\end{array}$ & {$[62]$} \\
\hline BSA-Lévy flight (LFBSA) & Simple & $\begin{array}{l}\mathrm{SD} \\
\mathrm{DD}\end{array}$ & $\begin{array}{l}57 \text { mm RTC France solar cell, PWP201 } \\
\text { photovoltaic panel }\end{array}$ & Yes & $-/ 50000$ & {$[63]$} \\
\hline
\end{tabular}

and the DD model to extract the seven parameters of the photovoltaic cell [31]. By using metaheuristic algorithms, all parameters of the photovoltaic cells and panels can be calculated. There are a lot of metaheuristic algorithms applied to extract the parameters of the photovoltaic cells and panels. The lower and upper values for the photovoltaic cells or panel parameters are necessary to be considered for the limitation of the global optimum search. Table 2 presents some of them, classified on family and on whether they are simple or hybrid [8]. The families of the algorithms presented are genetic algorithms (GA) [31-34], differential evolution (DE) [35-38], particle swarm optimization (PSO) [39-43], discretization [3, 44, 45], artificial bee colony (ABC) [46, 47], shuffled complex evolution [48, 49], simulated annealing (SA) $[50,51]$, flower pollination algorithm (FPA) [52, 53], harmony search (HS) [54], JAYA algorithm [55-57], teaching-learning-based optimization algorithm $[58,59]$, whale optimization algorithm $[60,61]$, and backtracking search algorithm $[62,63]$. Additionally, the diode model is shown, computational time and the iteration number when these are given.
One of the new and the best algorithms, HSDA [44], is used against the modified analytical method to forecast the $I-V$ characteristic and maximum power generated. The HSDA algorithm is an improved version of the SDA algorithm [3]. It is a hybrid one. The first algorithm used is one of the existent algorithms and gives a solution for SDA. A vicinity is considered around it, and the parameters can be extracted with very good accuracy using SDA for this vicinity. The flow chart of the HSDA algorithm is presented in Figure 2.

\section{Results and Discussion}

4.1. Analytical vs. Metaheuristic. The representative analytical method-modified five parameters-which has low difficulty to use and high accuracy, for SD model, it is compared with the best metaheuristic algorithms for each family, for several photovoltaic cells and panels when the results for the parameters are available. The parameter values for the SD model given in the references are used to calculate the statistical tests $\mathrm{AE}, \mathrm{RMSE}$, and $R^{2}$ used for comparison. 


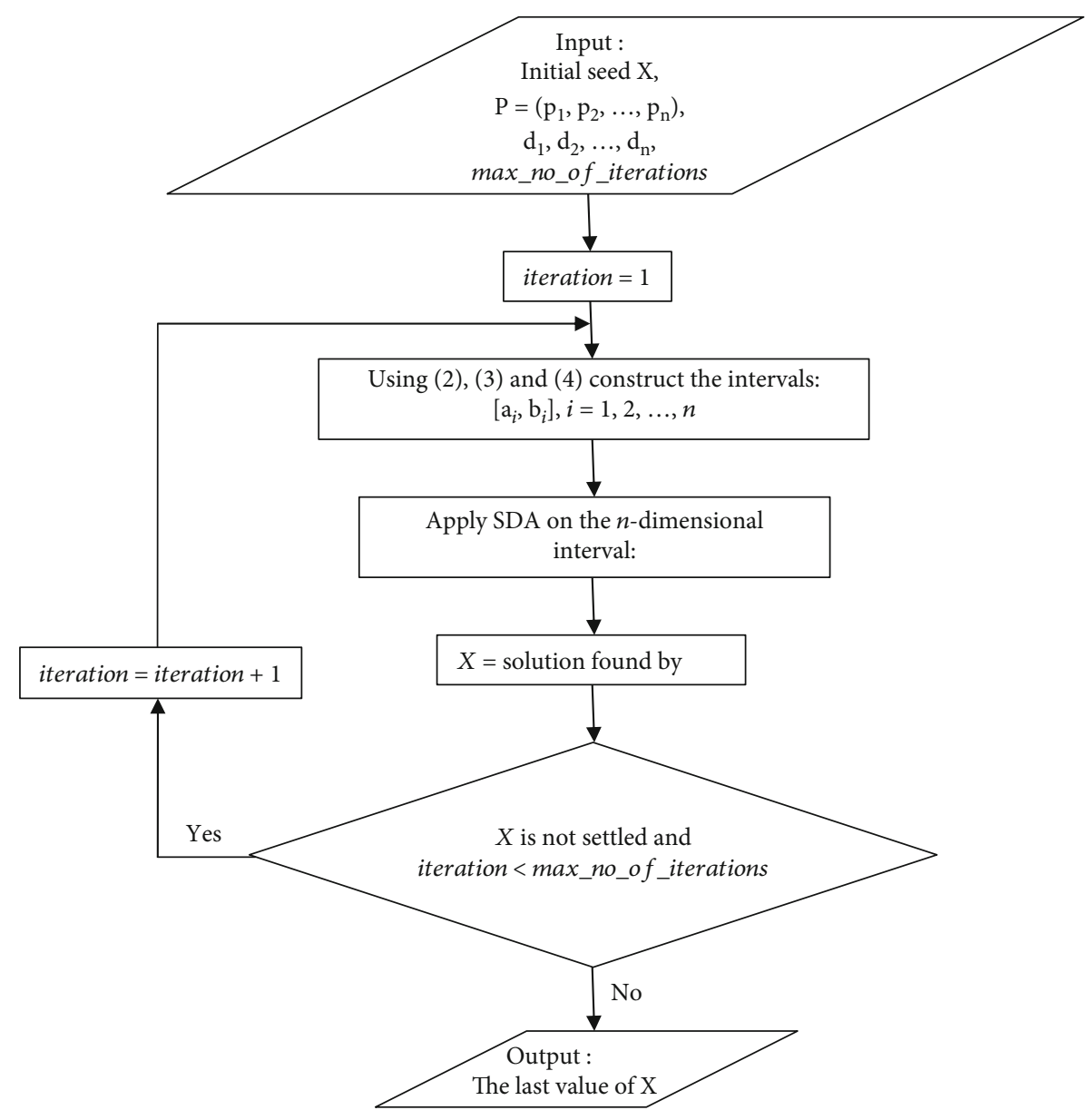

FIgURE 2: HSDA algorithm flow chart [44].

TABLE 3: The parameters and statistical tests for RTC cell.

\begin{tabular}{lcccccccc}
\hline Algorithm & $I_{\mathrm{ph}}(\mathrm{A})$ & $I_{\mathrm{o}}(\mu \mathrm{A})$ & $n$ & $R_{\mathrm{s}}(\Omega)$ & $R_{\mathrm{sh}}(\Omega)$ & $\mathrm{RMSE}$ & $\mathrm{AE}$ & $R^{2}$ \\
\hline HSDA [44] & 0.7607758 & 0.323016532 & 1.48118232 & 0.03637708 & 53.714520885 & $9.8602 E-04$ & 0.0215277 & 0.9999893 \\
$\mathrm{R}_{\mathrm{cr}}$-IJADE [36] & 0.76077553 & 0.3230208 & 1.4811836 & 0.03637709 & 53.718525 & $9.86021 E-4$ & 0.0215271 & 0.9999893 \\
C-HCLPSO [43] & 0.76079 & 0.31062 & 1.4771 & 0.036548 & 52.885 & $1.1201 E-03$ & 0.0209115 & 0.999986 \\
ABC-NMS [47] & 0.760776 & 0.323021 & 1.481184 & 0.036377 & 53.718521 & $9.86023 E-04$ & 0,021533 & 0.9999893 \\
ESCE-OBL [49] & 0.76078 & 0.32302 & 1.48118 & 0.03638 & 53.7185 & $9.8602 E-04$ & 0.0215269 & 0.9999893 \\
LM-SA [50] & 0.76078 & 0.31849 & 1.47976 & 0.03643 & 53.32644 & $9.8646 E-04$ & 0.0215104 & 0.9999892 \\
FPA [51] & 0.76079 & 0.31062 & 1.47707 & 0.03655 & 52.8771 & $1.214 E-03$ & 0.0216788 & 0.9999837 \\
IGHS [53] & 0.76077 & 0.34351 & 1.48740 & 0.03613 & 53.2845 & $1.033 E-03$ & 0.0212025 & 0.9999882 \\
CLJAYA [57] & 0.76078 & 0.3230208 & 1.481184 & 0.0363771 & 53.718521 & $9.8603 E-04$ & 0.0215415 & 0.9999892 \\
ITLBO [59] & 0.7608 & 0.3230 & 1.4812 & 0.0364 & 53.7185 & $9.9161 E-04$ & 0.021809 & 0.9999891 \\
IWOA [61] & 0.7608 & 0.3232 & 1.4812 & 0.0364 & 53.7317 & $9.9486 E-04$ & 0.021131 & 0.9999891 \\
MLBSA [62] & 0.7608 & 0.32302 & 1.4812 & 0.0364 & 53.7185 & $9.8969 E-04$ & 0.0217216 & 0.9999892 \\
5Pm [3] & 0.7612 & 0.1966 & 1.43 & 0.042 & 95.28 & $8.674 E-03$ & 0.159698 & 0.999086 \\
GA [33] & 0.7619 & 0.8087 & 1.5751 & 0.0299 & 42.3729 & 0.01908 & 0.277673 & 0.995997 \\
\hline
\end{tabular}




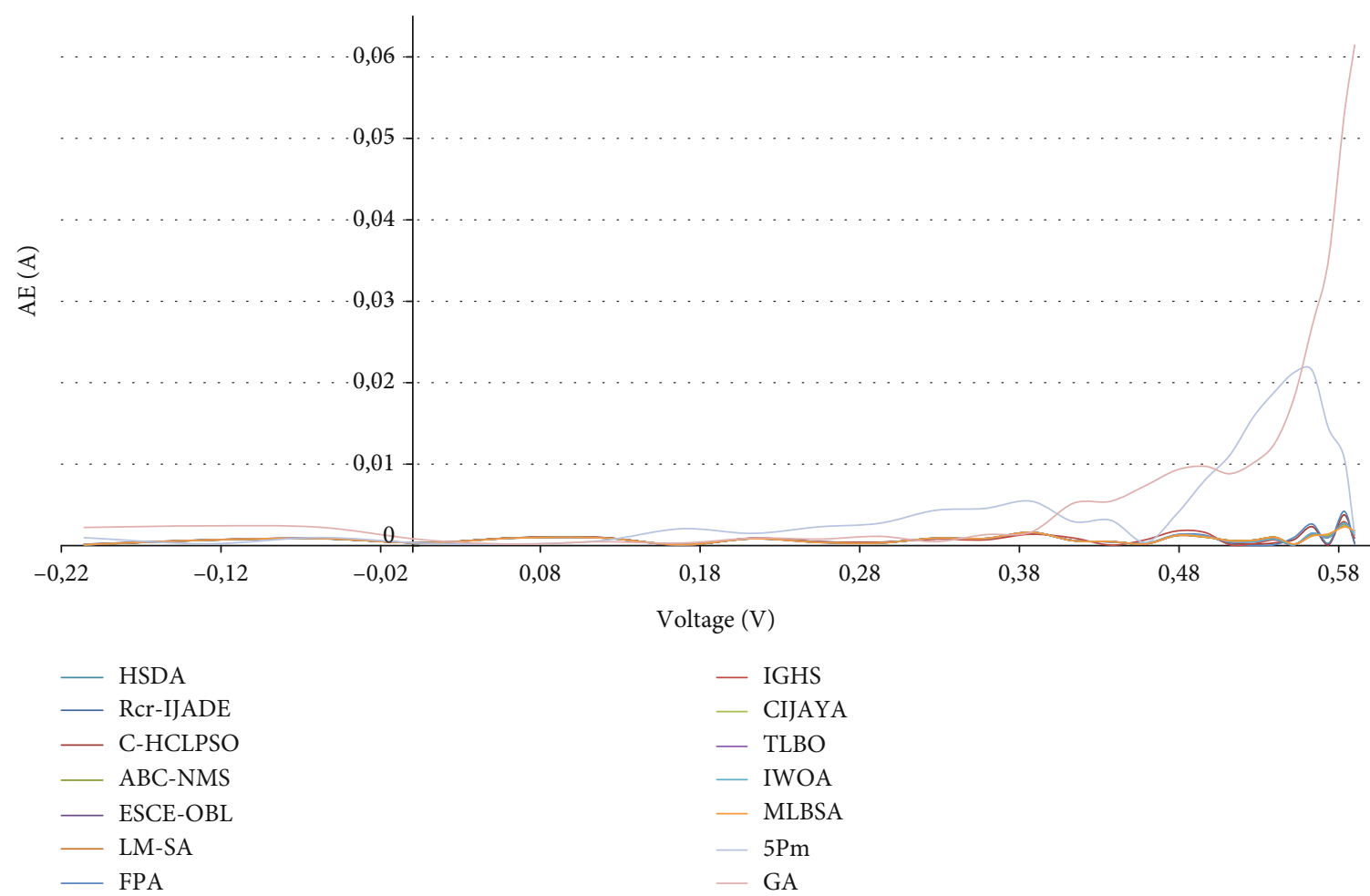

(a)

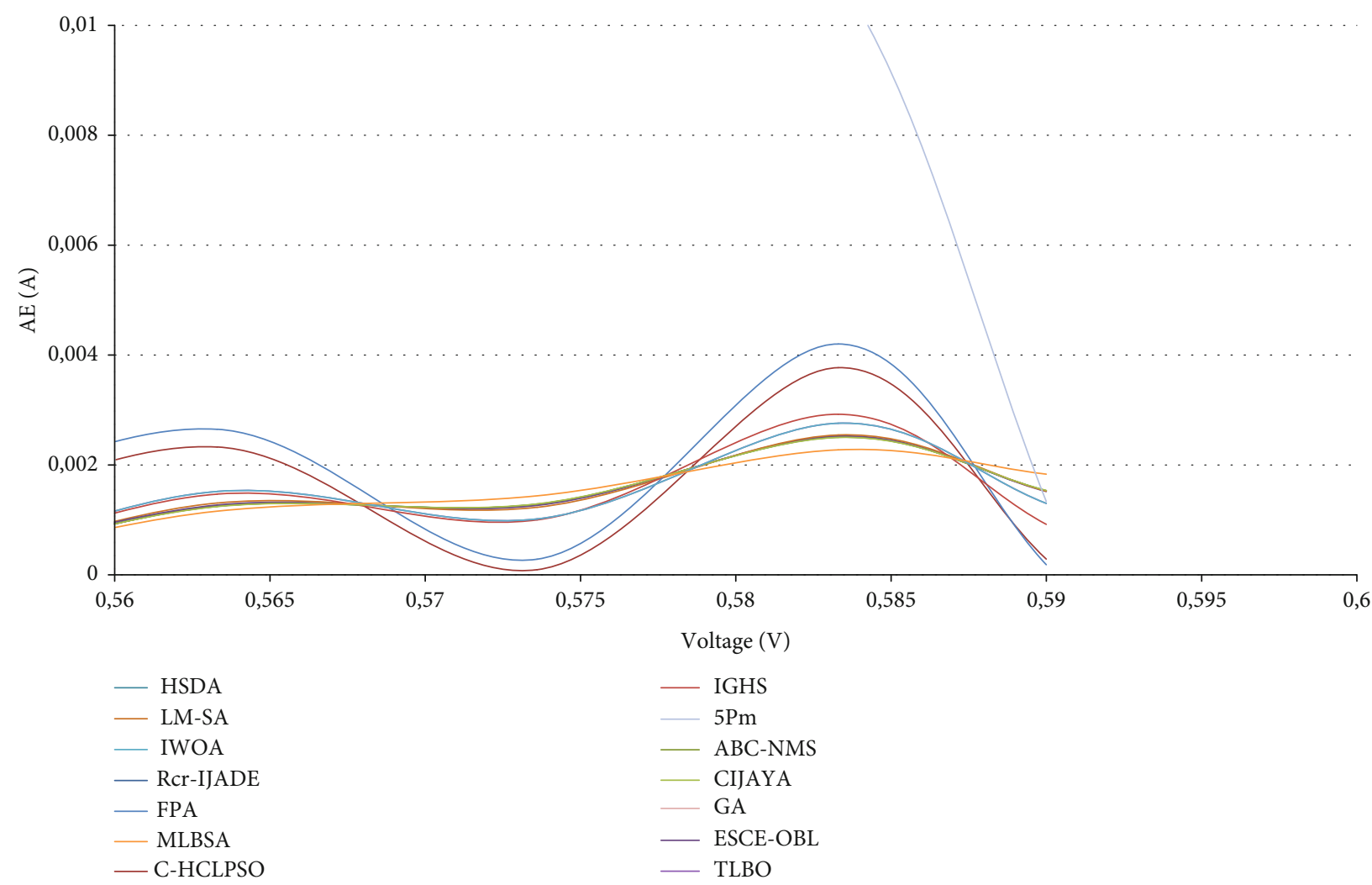

(b)

Figure 3: (a) The absolute current error (AE) for RTC France solar cell; (b) AE in open-circuit voltage region. 
TABLE 4: The parameters and statistical tests for mSi commercial photovoltaic cell.

\begin{tabular}{lcccccccc}
\hline Algorithm & $I_{\mathrm{ph}}(\mathrm{A})$ & $I_{\mathrm{o}}(\mu \mathrm{A})$ & $n$ & $R_{\mathrm{s}}(\Omega)$ & $R_{\mathrm{sh}}(\Omega)$ & $\mathrm{RMSE}$ & $\mathrm{AE}$ & $R^{2}$ \\
\hline HSDA [44] & 0.42575316 & 0.516241613 & 1.67933406 & 0.09132898 & 99.075980176 & $5.63098 E-04$ & 0.0142648 & 0.9999853 \\
5Pm [3] & 0.4255 & 0.30645567 & 1.618311 & 0.10352224 & 145.222 & $2.25639 E-03$ & 0.047492 & 0.99976 \\
GA [33] & 0.4256882 & 0.8383311 & 1.73926 & 0.0859435 & 123.3659 & $6.9741 E-04$ & 0.0163354 & 0.99997 \\
\hline
\end{tabular}

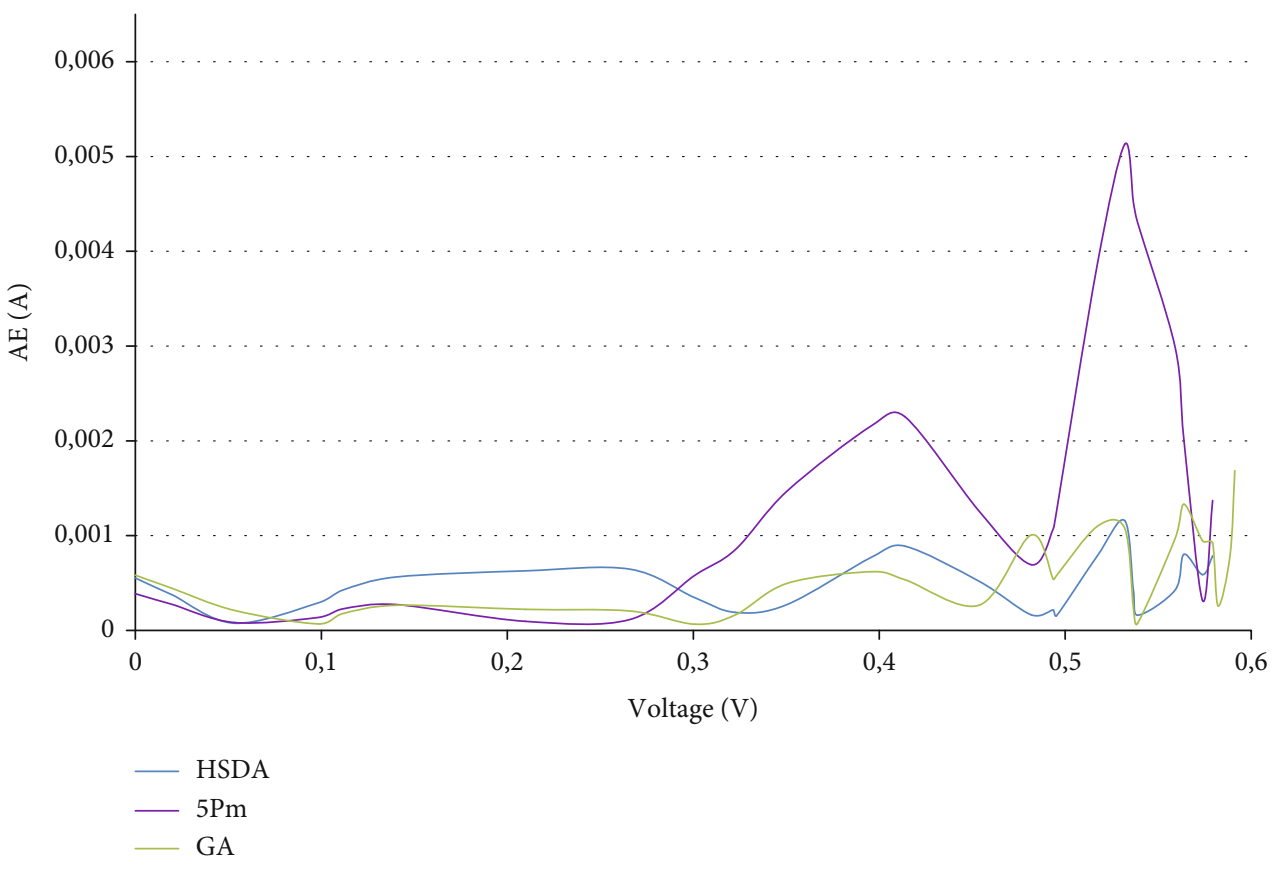

FIgURE 4: The absolute current error for $\mathrm{mSi}$ commercial photovoltaic cell.

TABle 5: The parameters and statistical tests for PWP201 photovoltaic panel.

\begin{tabular}{lcccccccc}
\hline Algorithm & $I_{\mathrm{ph}}(\mathrm{A})$ & $I_{\mathrm{o}}(\mu \mathrm{A})$ & $n$ & $R_{\mathrm{s}}(\Omega)$ & $R_{\mathrm{sh}}(\Omega)$ & $\mathrm{RMSE}$ & $\mathrm{AE}$ \\
\hline HSDA [44] & 1.0305143 & 0.348226304 & 48.642835 & 1.201271 & 981.201271 & $2.42507 E-03$ & 0.0489237 & 0.99997 \\
$\mathrm{R}_{\mathrm{cr}}$ IJADE [36] & 1.0305143 & 3.4822629 & 48.642835 & 1.201271 & 981.98216 & $2.42507 E-03$ & 0.0489237 & 0.99997 \\
ABC-NMS [47] & 1.03051 & 3.48226 & 48.643 & 1.20127 & 981.982 & $2.42518 E-03$ & 0.0489454 & 0.99997 \\
FPA [51] & 1.032091 & 3.047538 & 48.13128 & 1.217583 & 811.3721 & $2.57361 E-03$ & 0.0533746 & 0.99996 \\
CLJAYA [57] & 1.030514 & 3.4822628 & 48.64283 & 1.201271 & 981.982279 & $2.42507 E-03$ & 0.0489227 & 0.99997 \\
ITLBO [59] & 1.0305 & 3.4823 & 48.6428 & 1.2013 & 981.9823 & $2.42519 E-03$ & 0.0488878 & 0.99997 \\
IWOA [61] & 1.0305 & 3.4717 & 48.6313 & 1.2016 & 978.6771 & $2.42523 E-03$ & 0.0488933 & 0.99997 \\
MLBSA [62] & 1.0305 & 3.4823 & 48.6428 & 1.20163 & 981.9823 & $2.42561 E-03$ & 0.04878 & 0.99997 \\
5Pm [3] & 1.034 & 3.571 & 48.71 & 1.206 & 1123.00 & $4.019 E-03$ & 0.0833522 & 0.99991 \\
\hline
\end{tabular}

Additionally, the GA which will be used for forecast is considered.

4.1.1. RTC France Solar Cell. The first comparison is made for the RTC France solar cell, one of the widely used by researchers to prove the performance of the developed algorithms to extract the parameters of the photovoltaic cells and panels. The result of the parameters and the statistical tests, RMSE, AE, and $R^{2}$, are presented in Table 3.
In the case of the RTC France solar cell, the 5Pm method has the RMSE, which is widely used to measure the performance of the methods, higher than the ones obtained for the metaheuristic algorithms with the exception of the GA. Also, for the $\mathrm{AE}$ and $R^{2}$, the values are higher.

To have a complete image of the results obtained using different methods, Figure 3(a) presents the absolute current error. The 5Pm method and GA algorithm overestimate or underestimate the current around the open-circuit voltage 


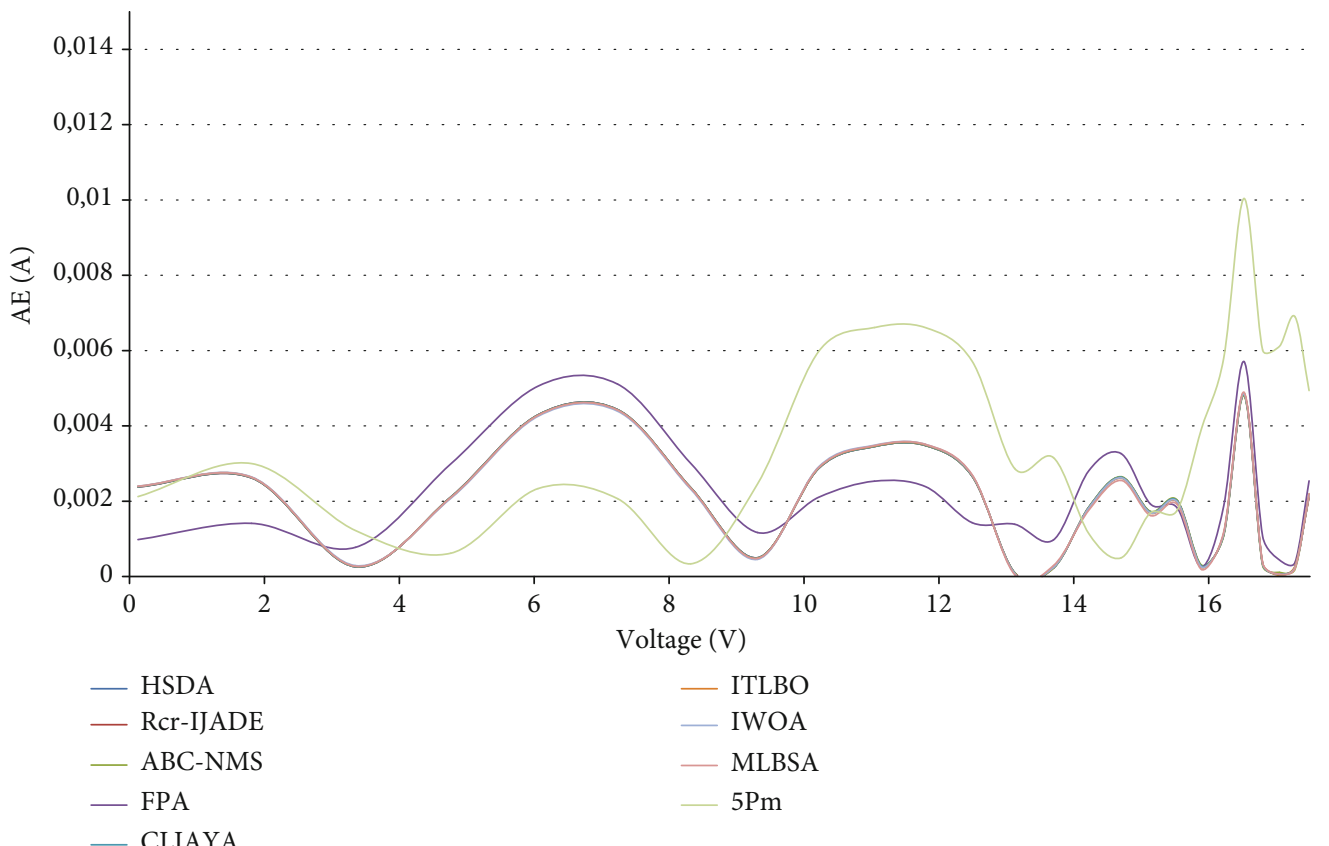

(a)

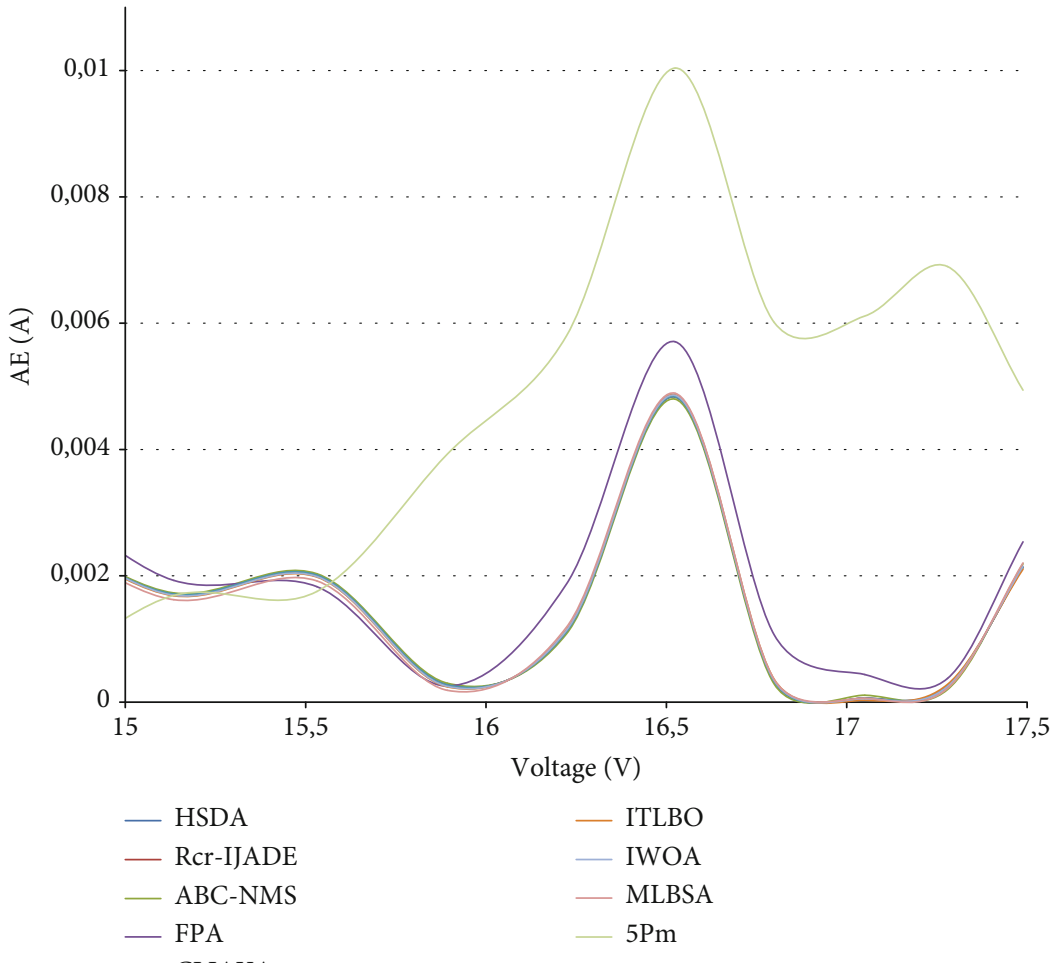

(b)

Figure 5: (a) The absolute current error (AE) for PWP201 photovoltaic panel; (b) AE in open-circuit voltage region.

point, where the other algorithms calculate the current better. The reverse saturation current extracted with the GA algorithm is more than two times higher than that calculated with HSDA. The parasitic resistances, the series resistance, and the shunt resistance present also a high variation. Figure 3(b) shows the behaviour of the $\mathrm{AE}$ around the open-circuit voltage region. The $\mathrm{AE}$ values for the $\mathrm{C}$ -
HCLPSO and FPA algorithms alternate around the AE average of the other algorithms considered, having high values for some regions and very small for other regions.

4.1.2. Commercial Monocrystalline Silicon Photovoltaic Cell. There are three methods to extract parameters of $\mathrm{mSi}$ commercial photovoltaic cell. The $5 \mathrm{Pm}$ analytical method gives 
TABLE 6: The parameters and statistical tests for the STM6-40 photovoltaic panel.

\begin{tabular}{lcccccccc}
\hline Algorithm & $I_{\mathrm{ph}}(\mathrm{A})$ & $I_{\mathrm{o}}(\mu \mathrm{A})$ & $n$ & $R_{\mathrm{s}}(\Omega)$ & $R_{\mathrm{sh}}(\Omega)$ & $\mathrm{RMSE}$ & $\mathrm{AE}$ & $R^{2}$ \\
\hline HSDA [44] & 1.6639047799 & 1.7386543978 & 54.730899 & 0.153855932 & 543.41834985 & $1.72981 E-03$ & 0.0219035 & 0.99997731 \\
$\mathrm{R}_{\mathrm{cr}}$ IJADE [36] & 1.6639 & 1.7387 & 54.7308 & 0.1548 & 573.4188 & $1.73428 E-03$ & 0.0216148 & 0.99997719 \\
ITLBO [59] & 1.6639 & 1.7387 & 54.7308 & 0.1548 & 573.4188 & $1.73428 E-03$ & 0.0216148 & 0.99997719 \\
5Pm [3] & 1.6636 & $2.6541 E-4$ & 33.3534 & 0.9121 & 898.16 & $3.53507 E-02$ & 0.540948 & 0.98999793 \\
\hline
\end{tabular}

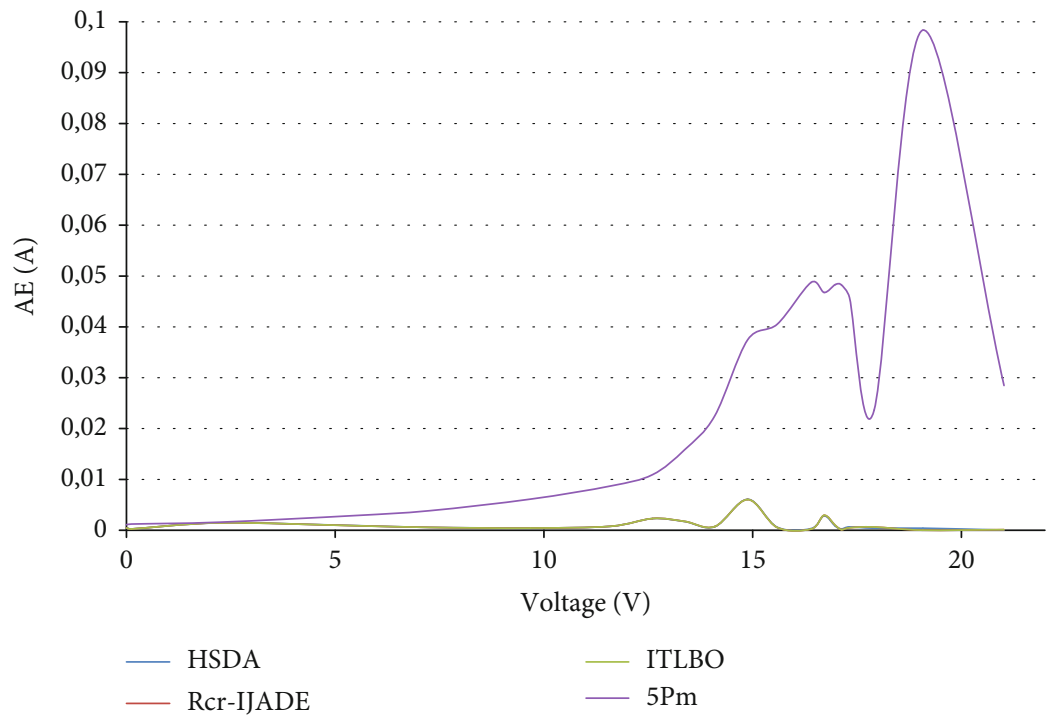

(a)

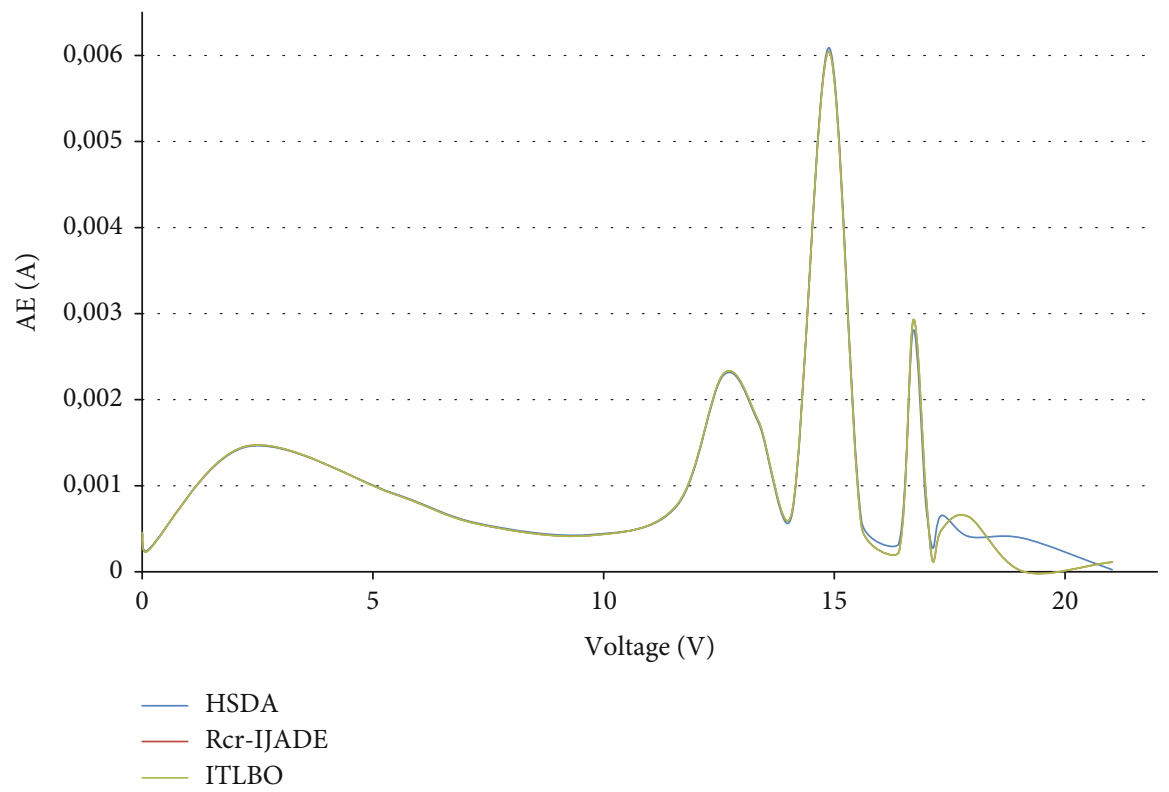

(b)

FIgURE 6: (a) The absolute current error (AE) for STM6-40 photovoltaic panel; (b) AE in open-circuit voltage region.

the weakest results for all statistical tests, Table 4. If in the case of the RTC photovoltaic cell, the RMSE obtained using the $5 \mathrm{Pm}$ method is almost ten times higher; for the mSi photovoltaic cell, the RMSE is 4.5 times higher, but the GA algorithm significantly improves its performance.
These changes in the performance of the methods can be easily observed in Figure 4 . The performance of the GA algorithm is substantially improved for the region around the open circuit point, while the 5Pm method shows weakness in this region. 
TABLE 7: The parameters and statistical tests for mSi photovoltaic panel.

\begin{tabular}{lcccccccc}
\hline Algorithm & $I_{\mathrm{ph}}(\mathrm{A})$ & $I_{\mathrm{o}}(\mu \mathrm{A})$ & $n$ & $R_{\mathrm{s}}(\Omega)$ & $R_{\mathrm{sh}}(\Omega)$ & $\mathrm{RMSE}$ & $\mathrm{AE}$ & $R^{2}$ \\
\hline HSDA [44] & 1.224206 & $4.677 E-4$ & 18.364994 & 0.14407 & 1544.361724 & $2.77734 E-03$ & 1.93926 & 0.99956777 \\
GA [33] & 1.223082 & $4.988143 E-3$ & 20.5289 & 0.02147292 & 1765.388 & $4.96271 E-03$ & 2.61456 & 0.9984 \\
5Pm [3] & 1.224 & $0.334 E-3$ & 18.02 & 0.134 & 1242.91 & $6.21773 E-03$ & 2.42416 & 0.9978 \\
\hline
\end{tabular}

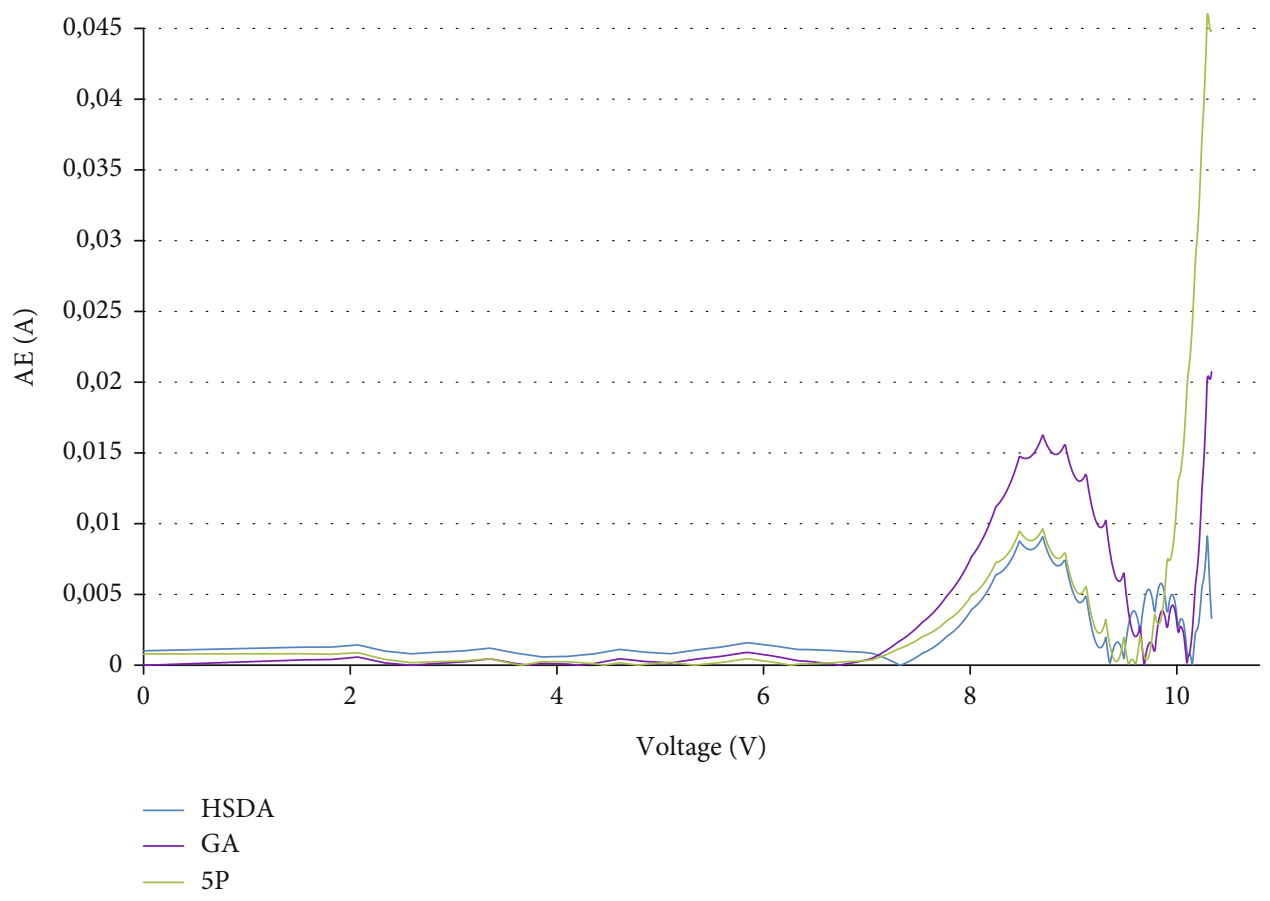

Figure 7: The absolute current error for $\mathrm{mSi}$ photovoltaic panel.

4.1.3. PWP201 Photovoltaic Panel. Analyzing the results obtained for RMSE in the case of the PWP201 photovoltaic panel, RMSE and AE obtained with the 5Pm method are 1.65 times higher than the ones obtained with the HSDA algorithm. There are three algorithms with the best values for all three statistical tests, HSDA, $\mathrm{R}_{\mathrm{cr}}$-IJADE, and CLJAYA, Table 5.

The absolute current errors for PWP201 photovoltaic panel are under 0.01 (A), having a uniform distribution, but keeping the high values in the open-circuit voltage region, Figure 5(a). Although, in this case, the methods estimate the current without high difference for certain voltages in comparison with the ones measured, the RMSE and AE have high values. The AE for the PWP201 photovoltaic panel around the open-circuit voltage is higher for the FPA and $5 \mathrm{Pm}$ methods. The other algorithms considered have the same behaviour, Figure 5(b).

4.1.4. STM6-40 Photovoltaic Panel. The 5Pm method has the statistical test high values, Table 6 . The $(V, I)$ pairs of the STM6-40 photovoltaic panel are not uniformly distributed. There are very few points in the open-circuit voltage region [46], which leads to poorer results in this case. The value of the coefficient of determination confirms this issue.
TABLE 8: The statistical tests for the forecast $I-V$ characteristic.

\begin{tabular}{lcc}
\hline Algorithm & RMSE $f$ & RMSE $m$ \\
\hline HSDA [44] & $5.30433 E-03$ & $3.234 E-03$ \\
GA [33] & $1.63618 E-02$ & $6.707 E-03$ \\
5Pm [3] & $6.23956 E-03$ & $2.86 E-02$ \\
\hline
\end{tabular}

The best results are obtained for the HSDA algorithm for RSME and $R^{2}$. The plot of the absolute current errors, Figure 6(a), shows the weakness of the 5Pm method in the region around the open-circuit voltage. For some points, the current calculated with the $5 \mathrm{Pm}$ method is twenty times higher than the ones calculated with the HSDA algorithm.

The behaviour of the AE around the open-circuit voltage is similar for the ITLBO and the $\mathrm{R}_{\mathrm{cr}}$-IJADE algorithms, Figure 6(b). The AE for the HSDA algorithm has some small variations.

4.1.5. Commercial Monocrystalline Silicon Photovoltaic Panel. The statistical tests for commercial mSi photovoltaic panel are presented in Table 7.

The shape of the absolute current error curves is the same in the case of the mSi photovoltaic panel. The highest 


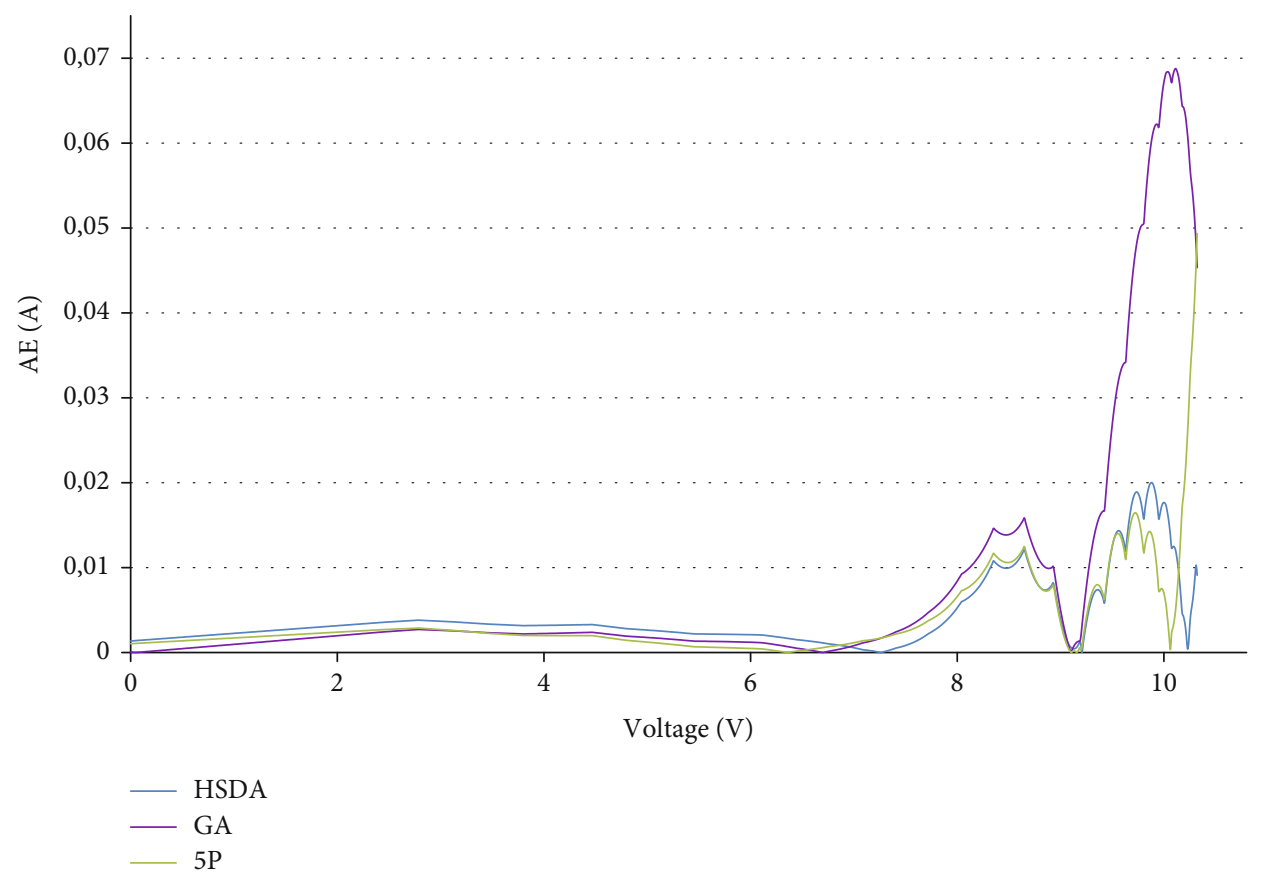

(a)

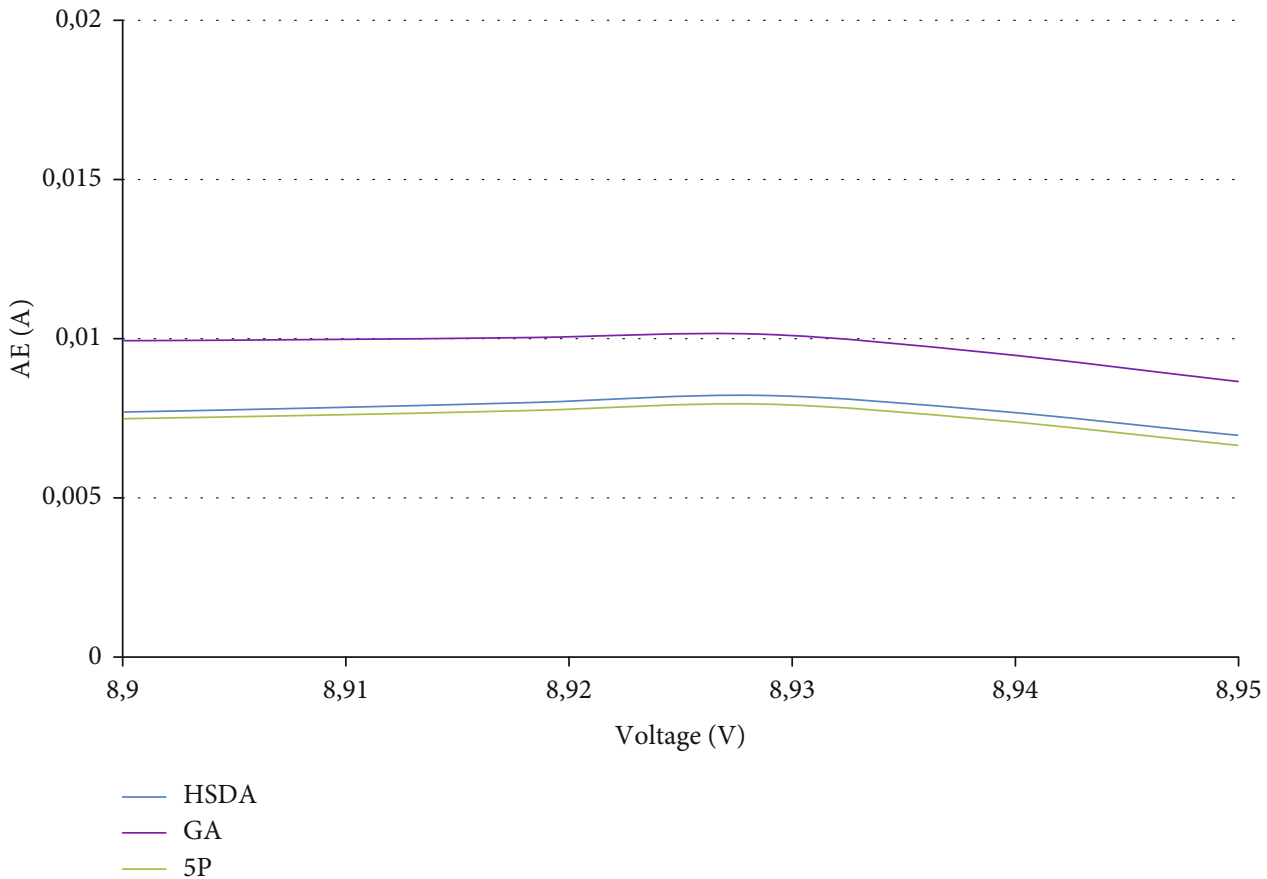

(b)

Figure 8: (a) The absolute current error (AE) for the forecasted $I$ - $V$ characteristic for photovoltaic panel; (b) AE in the voltage which corresponds to maximum power region.

value for the $\mathrm{AE}$ is again in the region of the open-circuit voltage, Figure 7.

The coefficient of determination for all photovoltaic cells and panels is very good, less for the GA algorithm in case of RTC photovoltaic cell and STM6-40 photovoltaic panel.

4.2. Forecast Comparison. Two $I-V$ characteristics are measured for the commercial monocrystalline photovoltaic
TABle 9: The $P_{\max }$ forecasted and measured.

\begin{tabular}{lcccc}
\hline Algorithm & HSDA [44] & GA [33] & 5Pm [3] & Measured \\
\hline$P_{\max }(\mathrm{W})$ & 13.807 & 13.795 & 13.809 & 13.875 \\
$P_{\max , f} / P_{\max , m}(\%)$ & 99.509 & 98.45 & 99.52 & \\
\hline
\end{tabular}


TABle 10: Advantages and disadvantages.

\begin{tabular}{|c|c|c|}
\hline Method & Advantages & Disadvantages \\
\hline Analytical & $\begin{array}{l}\text { (i) Using the } 5 \mathrm{Pm} \text { method is very easy, the } R_{\mathrm{so}} \text { and } R_{\mathrm{sh}} \text { are } \\
\text { calculated with the empirical equations [25] } \\
\text { (ii) Very easy to implement on the computer for all software } \\
\text { which allows calculation } \\
\text { (iii) The necessary time to extract the parameters is very } \\
\text { short for the 5Pm method (duration is under ms) } \\
\text { (iv) Does not require a powerful or dedicated PC, which } \\
\text { leads to the small cost of the PC, and the used software can } \\
\text { be a common one }\end{array}$ & $\begin{array}{l}\text { (i) The accuracy of the parameters extracted decreases due to } \\
\text { approximations used [8] } \\
\text { (ii) If some points of the } I-V \text { characteristic are used, the } \\
\text { equation system obtained is nonlinear and it is necessary to } \\
\text { use software that allows solving this (this can be costly) } \\
\text { (iii) The complexity increases when the two diode model is } \\
\text { used (seven equations are needed) }\end{array}$ \\
\hline Metaheuristic & $\begin{array}{l}\text { (i) The accuracy is higher than that of the analytical methods } \\
\text { (ii) The number of iterations can be reduced (for example, } \\
\text { for the ISCE algorithm, the iteration number is 5000) } \\
\text { (iii) The algorithms can be easily adapted for the two diode } \\
\text { model, but the computational time will increase } \\
\text { (iv) Using algorithms as grey wolf, prey predator, and fire fly } \\
\text { optimization, the performance of the parameters extraction } \\
\text { can be improved [8] } \\
\text { (v) GA algorithm family can easy be part of the hybrid } \\
\text { algorithm [65] } \\
\text { (vi) DE algorithm family has a high convergence } \\
\text { (vii) PSO can be improved if it is used together with Nelder- } \\
\text { Mead methods, this hybridisation can reduce the } \\
\text { computational resources [65] } \\
\text { (viii) SDA family has very good performance, and it can be } \\
\text { easily used with other algorithms in hybrid structure } \\
\text { (ix) Improved version of SCE has performance comparable } \\
\text { with HSDA }\end{array}$ & $\begin{array}{l}\text { (i) The computational time can be high, it depends on the } \\
\text { PC power and the metaheuristic algorithm (the number of } \\
\text { the iterations and the complexity), there are algorithms with } \\
\text { over } 100000 \text { iterations or even } 350000 \text {-NM-MPSO } \\
\text { algorithm [41] } \\
\text { (ii) The cost of the PC can be high and the dedicated } \\
\text { software must be used } \\
\text { (iii) The performance of the most metaheuristic algorithms } \\
\text { depend by the initial parameter range } \\
\text { (iv) Requires very good knowledge for implementation } \\
\text { (v) GA, SCE, TLBO, WOA, and BSA algorithms have a slow } \\
\text { convergence } \\
\text { (vi) DE and PSO algorithms can converge prematurely } \\
\text { (vii) The computational time is high in case of the PSO } \\
\text { algorithms } \\
\text { (viii) The computational time is relatively high, but using } \\
\text { parallelization, it can be reduced; in the case presented, the } \\
\text { time was reduced } 6 \text { times }\end{array}$ \\
\hline
\end{tabular}

panel for the irradiance of $700 \mathrm{~W} / \mathrm{m}^{2}$ and $983 \mathrm{~W} / \mathrm{m}^{2}$. The measurements were made on the roof of the university building [3].

The $I-V$ characteristic measured at $700 \mathrm{~W} / \mathrm{m}^{2}$ was used to extract the parameters of the $\mathrm{mSi}$ photovoltaic panel with three methods HSDA algorithm, GA algorithm, and 5Pm analytical method. These parameters are considered as $I_{\text {ph,ref }}, I_{\text {o,ref }}, n_{\text {ref }}, R_{\mathrm{s}, \text { ref }}$, and $R_{\text {sh,ref }}$. The parameters of the $\mathrm{mSi}$ photovoltaic panel are calculated for $983 \mathrm{~W} / \mathrm{m}^{2}$ irradiance, using Equations (7)-(11). These values are used to compare the measured $I-V$ characteristic for $983 \mathrm{~W} / \mathrm{m}^{2}$ in real conditions with the ones forecasted with the five parameters calculated with Equations (7)-(11). The temperature coefficient of the current was determined, and it is $15.76 \mathrm{~mA} /{ }^{\circ} \mathrm{C}$.

The root mean square error for the forecasted $I-V$ characteristic of the $\mathrm{mSi}$ photovoltaic panel is presented in Table 8 in comparison with one for the $I-V$ characteristic measured. The RMSE $f$ obtained when the parameters are calculated with Equations (7)-(11) is worse in the case of the HSDA and GA algorithms than the RMSE $m$ obtained using the extracted parameters with those algorithms for the measured characteristic. In case of the 5Pm methods, the results are improved.

The absolute current error calculated for the forecast $I-V$ characteristic in comparison with the ones measured is presented in Figure 8(a). The highest values for AE are in the region of the open-circuit voltage. The behaviour of the curves obtained with parameters calculated with Equations (7)-(11) for HSDA and 5Pm methods is very similar, while the ones obtained with GA algorithms have an accentuated increase in the open-circuit voltage region.

Figure $8(\mathrm{~b})$ shows the behaviour of the absolute current error around the maximum voltage, $V_{m}$, which is the voltage coordinate for the maximum power point. The best results are obtained for the $5 \mathrm{Pm}$ method for all regions considered.

The comparison between maximum power generated, $P_{\max , m}$, the $\mathrm{mSi}$ panel calculated from real measurements and ones forecasted, $P_{\max , f}$, using the extracted parameters with the three methods, Table 9, shows that the best estimation is for the maximum power estimated with parameters extracted with the $5 \mathrm{Pm}$ method.

By analyzing the results obtained through comparison between the analytic method and metaheuristic algorithms, it can be concluded that the 5Pm method can be used to extract the parameters of the photovoltaic cells and panels. Additionally, the analytical method can be used to estimate the $I-V$ characteristics and the power generated using the parameters given by the producers. It can be used due to the advantages which are presented in Table 10.

\section{Conclusions}

The paper briefly reviews the analytical methods and metaheuristic algorithms used to extract the five or seven parameters for the photovoltaic cells and panels. The 5Pm 
analytical method and ones of the best metaheuristic algorithms from different families are compared for five datasets, two for photovoltaic cells, and three for photovoltaic panels.

By analyzing the results obtained, the supremacy of the metaheuristic algorithms for accuracy is shown. In all cases studied, the algorithms have better results for all statistical tests used. The analytical method has a better performance than the GA algorithm for the RTC photovoltaic cell. The performance of the HSDA algorithm is one of the best for all photovoltaic cells and panels analyzed, and it was chosen to be compared in the forecast process.

Two $I-V$ characteristics, measured for the commercial $\mathrm{mSi}$ photovoltaic panel, are used to compare the influence of the extract parameter methods on the forecast of the maximum power and $I-V$ characteristic at different values of the irradiance and temperature. Using the parameters calculated with the HSDA algorithm, the forecast of the $I-V$ characteristic was better than for GA and 5Pm methods. However, the $5 \mathrm{Pm}$ method forecasted better the maximum power, only $0.48 \%$ less than the real one. These are preliminary results, which will further be developed in future research by analysis for various cases. This analysis will be made on different panels, under various irradiance and illumination conditions.

The 5Pm methods are based on several relations which are easy to implement, for the measured $I-V$ characteristic, datasets, or the datasheet parameters, the latter offered by the producer, and the parameters can be quickly calculated with very good accuracy. The necessary time to calculate the parameters is very small, and it does not require a powerful PC, as for the metaheuristic algorithms. These prove that the $5 \mathrm{Pm}$ method is a valuable candidate for photovoltaic cells and panel manufacturers. They can use the $5 \mathrm{Pm}$ method to characterize the photovoltaic devices and to obtain the optimum photovoltaic panels using cells with the same values of the parameters. The production time and the costs can be optimized.

\section{Conflicts of Interest}

The authors declare that there is no conflict of interest regarding the publication of this paper.

\section{Acknowledgments}

This work was supported by a grant of the Romanian Ministry of Education and Research, CCCDI-UEFISCDI, project number PN-III-P2-2.1-PED-2019-2028, within PNCDI III.

\section{References}

[1] D. T. Cotfas, P. A. Cotfas, and S. Kaplanis, "Methods to determine the dc parameters of solar cells: a critical review," Renewable and Sustainable Energy Reviews, vol. 28, pp. 588-596, 2013.

[2] A. M. Humada, M. Hojabri, S. Mekhilef, and H. M. Hamada, "Solar cell parameters extraction based on single and doublediode models: a review," Renewable and Sustainable Energy Reviews, vol. 56, pp. 494-509, 2016.
[3] D. T. Cotfas, A. M. Deaconu, and P. A. Cotfas, "Application of successive discretization algorithm for determining photovoltaic cells parameters," Energy Conversion and Management, vol. 196, pp. 545-556, 2019.

[4] D. Sera, R. Teodorescu, and P. Rodriguez, "PV panel model based on datasheet values," in 2007 IEEE International Symposium on Industrial Electronics, pp. 2392-2396, Vigo, Spain, 2007.

[5] J. P. Ram, H. Manghani, D. S. Pillai, T. S. Babu, M. Miyatake, and N. Rajasekar, "Analysis on solar PV emulators: a review," Renewable and Sustainable Energy Reviews, vol. 81, pp. 149160, 2018.

[6] G. Huang, Y. Liang, X. Sun, C. Xu, and F. Yu, "Analyzing SShaped_I_-_V_characteristics of solar cells by solving threediode lumped-parameter equivalent circuit model explicitly," Energy, vol. 212, p. 118702, 2020.

[7] A. Elkholy and A. A. Abou El-Ela, "Optimal parameters estimation and modelling of photovoltaic modules using analytical method," Heliyon, vol. 5, no. 7, article e02137, 2019.

[8] D. S. Pillai and N. Rajasekar, "Metaheuristic algorithms for PV parameter identification: a comprehensive review with an application to threshold setting for fault detection in PV systems," Renewable and Sustainable Energy Reviews, vol. 82, no. 3, pp. 3503-3525, 2018.

[9] J. Bai, S. Liu, Y. Hao, Z. Zhang, M. Jiang, and Y. Zhang, "Development of a new compound method to extract the five parameters of PV modules," Energy Conversion and Management, vol. 79, pp. 294-79303, 2014.

[10] M. Kumar and A. Kumar, "An efficient parameters extraction technique of photovoltaic models for performance assessment," Solar Energy, vol. 158, pp. 192-206, 2017.

[11] D. T. Cotfas, P. A. Cotfas, and O. M. Machidon, "Study of temperature coefficients for parameters of photovoltaic cells," vol. 2018, 2018International Journal of Photoenergy, 2018.

[12] B. Van Zeghbroeck, Principles of Semiconductor Devices and Heterojunctions, Prentice Hall, Englewood Cliffs, New Jersey, 1 st edition, 2008.

[13] M. Wolf and H. Rauschenbach, "Series resistance effects on solar cell measurements," Advanced Energy Conversion, vol. 3, no. 2, pp. 455-479, 1963.

[14] A. Virtuani, E. Lotter, and M. Powalla, "Performance of $\mathrm{Cu}(\mathrm{In}, \mathrm{Ga}) \mathrm{Se}_{2}$ solar cells under low irradiance," Thin Solid Films, vol. 431-432, pp. 443-447, 2003.

[15] S. Nesmachnow, "An overview of metaheuristics: accurate and efficient methods for optimisation," International J. Metaheuristics, vol. 3, no. 4, pp. 320-347, 2014.

[16] D. S. H. Chan, J. R. Phillips, and J. C. H. Phang, "A comparative study of extraction methods for solar cell model parameters," Solid-State Electronics, vol. 29, no. 3, pp. 329-337, 1986.

[17] M. Tivanov, A. Patryn, N. Drozdov, A. Fedotov, and A. Mazanik, "Determination of solar cell parameters from its current-voltage and spectral characteristics," Solar Energy Materials and Solar Cells, vol. 87, no. 1-4, pp. 457-465, 2005.

[18] A. Ortiz-Conde, F. J. G. Sánchez, and J. Muci, "New method to extract the model parameters of solar cells from the explicit analytic solutions of their illuminated_I -V_ characteristics," Solar Energy Materials and Solar Cells, vol. 90, no. 3, pp. 352-361, 2006.

[19] C. L. Garrido-Alzar, "Algorithm for extraction of solar cell parameters from I-V curve using double exponential model," Renewable Energy, vol. 10, no. 2-3, pp. 125-128, 1997. 
[20] B. Arcipiani, "Generalization of the area method for the determination of the parameters of a non-ideal solar cell," Revue de Physique Appliquée, vol. 20, no. 5, pp. 269-272, 1985.

[21] G. L. Araujo and E. Sanchez, "A new method for experimental determination of the series resistance of a solar cell," IEEE Transactions on Electron Devices, vol. 29, no. 10, pp. 15111513, 1982.

[22] A. Kaminski, J. J. Marchand, A. Fave, and A. Laugier, "New method of parameters extraction from dark I-V curve," in Conference Record of the Twenty Sixth IEEE Photovoltaic Specialists Conference - 1997, pp. 203-206, Anaheim, CA, USA, 1997.

[23] N. N. Ulapane, C. H. Dhanapala, S. M. Wickramasinghe, S. G. Abeyratne, N. Rathnayake, and P. J. Binduhewa, "Extraction of parameters for simulating photovoltaic panels," in 2011 6th International Conference on Industrial and Information Systems, pp. 539-544, Kandy, Sri Lanka, 2011, IEEE.

[24] F. Ghani and M. Duke, "Numerical determination of parasitic resistances of a solar cell using the Lambert W-function," Solar Energy, vol. 85, no. 9, pp. 2386-2394, 2011.

[25] O. Mares, M. Paulescu, and V. Badescu, "A simple but accurate procedure for solving the five-parameter model," Energy Conversion and Management, vol. 105, pp. 139-148, 2015.

[26] R. Ndegwa, J. Simiyu, E. Ayieta, and N. Odero, “A Fast and accurate analytical method for parameter determination of a photovoltaic system based on manufacturer's data," Journal of Renewable Energy, vol. 2020, 7580218 pages, 2020.

[27] A. Hali and Y. Khlifi, "Photovoltaic panel parameters determination using two numerical methods," Materials Today: Proceedings, vol. 45, pp. 7377-7382, 2021.

[28] V. Lo Brano and G. Ciulla, "An efficient analytical approach for obtaining a five parameters model of photovoltaic modules using only reference data," Applied Energy, vol. 111, pp. 894903, 2013.

[29] A. Orioli and A. Di Gangi, "A criterion for rating the usability and accuracy of the one-diode models for photovoltaic modules," Energies, vol. 9, no. 6, p. 427, 2016.

[30] A. M. Humada, S. Y. Darweesh, K. G. Mohammed et al., "Modeling of PV system and parameter extraction based on experimental data: review and investigation," Solar Energy, vol. 199, pp. 742-760, 2020.

[31] J. A. Jervase, H. Bourdoucen, and A. Al-Lawati, "Solar cell parameter extraction using genetic algorithms," Measurement Science and Technology, vol. 12, no. 11, pp. 1922-1925, 2001.

[32] M. Zagrouba, A. Sellami, M. Bouaicha, and M. Ksouri, "Identification of PV solar cells and modules parameters using the genetic algorithms: application to maximum power extraction," Solar Energy, vol. 84, no. 5, pp. 860-866, 2010.

[33] D. T. Cotfas, P. A. Cotfas, and A. Cataron, "Using the genetic algorithm to determine the parameters of photovoltaic cells and panels," in 2018 International Symposium on Electronics and Telecommunications (ISETC), pp. 1-4, Timisoara, Romania, November 2018.

[34] X. Lingyun, S. Lefei, H. Wei, and J. Cong, "Solar cells parameter extraction using a hybrid genetic algorithm," in 2011 Third International Conference on Measuring Technology and Mechatronics Automation, pp. 306-309, Shanghai, China, 2011.

[35] X. Yang, W. Gong, and L. Wang, "Comparative study on parameter extraction of photovoltaic models via differential evolution," Energy Conversion and Management, vol. 201, p. 112113, 2019.
[36] W. Gong and Z. Cai, "Parameter extraction of solar cell models using repaired adaptive differential evolution," Solar Energy, vol. 94, pp. 209-220, 2013.

[37] K. Ishaque, Z. Salam, S. Mekhilef, and A. Shamsudin, "Parameter extraction of solar photovoltaic modules using penaltybased differential evolution," Applied Energy, vol. 99, pp. 297-308, 2012.

[38] D. H. Muhsen, A. B. Ghazali, T. Khatib, and I. A. Abed, "Parameters extraction of double diode photovoltaic module's model based on hybrid evolutionary algorithm," Energy Conversion and Management, vol. 105, pp. 552-561, 2015.

[39] H. Wei, J. Cong, X. Lingyun, and S. Deyun, "Extracting solar cell model parameters based on chaos particle swarm algorithm," in 2011 International Conference on Electric Information and Control Engineering, pp. 398-402, Wuhan, China, 2011.

[40] V. Khanna, B. K. Das, D. Bisht, Vandana, and P. K. Singh, “A three diode model for industrial solar cells and estimation of solar cell parameters using PSO algorithm," Renewable Energy, vol. 78, pp. 105-113, 2015.

[41] N. F. A. Hamid, N. A. Rahim, and J. Selvaraj, "Solar cell parameters identification using hybrid Nelder-Mead and modified particle swarm optimization," Journal of Renewable and Sustainable Energy, vol. 8, no. 1, 2016.

[42] D. Yousri, S. B. Thanikanti, D. Allam, V. K. Ramachandaramurthy, and M. Eteiba, "Fractional chaotic ensemble particle swarm optimizer for identifying the single, double, and three diode photovoltaic models' parameters," Energy, vol. 195, p. 116979, 2020.

[43] D. Yousri, D. Allam, M. Eteiba, and P. N. Suganthan, "Static and dynamic photovoltaic models' parameters identification using Chaotic Heterogeneous Comprehensive Learning Particle Swarm Optimizer variants," Energy Conversion and Management, vol. 182, pp. 546-563, 2019.

[44] D. T. Cotfas, A. M. Deaconu, and P. A. Cotfas, "Hybrid successive discretisation algorithm used to calculate parameters of the photovoltaic cells and panels for existing datasets," IET Renewable Power Generation, pp. 1-27, 2021.

[45] A. M. Deaconu, D. T. Cotfas, and P. A. Cotfas, "Calculation of seven photovoltaic cells parameters using parallelized successive discretization algorithm," International Journal of Photoenergy, vol. 2020, Article ID 6669579, 13 pages, 2020.

[46] D. Oliva, E. Cuevas, and G. Pajares, "Parameter identification of solar cells using artificial bee colony optimization," Energy, vol. 72, pp. 93-102, 2014.

[47] Z. Chen, L. Wu, P. Lin, Y. Wu, and S. Cheng, "Parameters identification of photovoltaic models using hybrid adaptive Nelder- Mead simplex algorithm based on eagle strategy," Applied Energy, vol. 182, pp. 47-57, 2016.

[48] X. Gao, Y. Cui, J. Hu et al., "Parameter extraction of solar cell models using improved shuffled complex evolution algorithm," Energy Conversion and Management, vol. 157, pp. 460-479, 2018.

[49] Y. Chen, Z. Chen, L. Wu, C. Long, P. Lin, and S. Cheng, "Parameter extraction of PV models using an enhanced shuffled complex evolution algorithm improved by oppositionbased learning," Energy Procedia, vol. 158, pp. 991-997, 2019.

[50] F. Dkhichi, B. Oukarfi, A. Fakkar, and N. Belbounaguia, "Parameter identification of solar cell model using Levenberg-Marquardt algorithm combined with simulated annealing," Solar Energy, vol. 110, pp. 781-788, 2014. 
[51] D. F. Alam, D. A. Yousri, and M. B. Eteiba, "Flower pollination algorithm based solar PV parameter estimation," Energy Conversion and Management, vol. 101, pp. 410-422, 2015.

[52] J. P. Ram, T. S. Babu, T. Dragicevic, and N. Rajasekar, “A new hybrid bee pollinator flower pollination algorithm for solar PV parameter estimation," Energy Conversion and Management, vol. 135, pp. 463-476, 2017.

[53] A. Askarzadeh and A. Rezazadeh, "Parameter identification for solar cell models using harmony search-based algorithms," Solar Energy, vol. 86, no. 11, pp. 3241-3249, 2012.

[54] M. F. AlHajri, K. M. el-Naggar, M. R. AlRashidi, and A. K. alOthman, "Optimal extraction of solar cell parameters using pattern search," Renewable Energy, vol. 44, pp. 238-245, 2012.

[55] K. Yu, J. Liang, B. Qu, X. Chen, and H. Wang, "Parameters identification of photovoltaic models using an improved JAYA optimization algorithm," Energy Conversion and Management, vol. 150, pp. 742-753, 2017.

[56] K. Yu, B. Qu, C. Yue, S. Ge, X. Chen, and J. Liang, "A performance-guided JAYA algorithm for parameters identification of photovoltaic cell and module," Applied Energy, vol. 237, pp. 241-257, 2019.

[57] Y. Zhang, M. Ma, and Z. Jin, "Comprehensive learning Jaya algorithm for parameter extraction of photovoltaic models," Energy, vol. 211, p. 118644, 2020.

[58] S. J. Patel, A. K. Panchal, and V. Kheraj, "Extraction of solar cell parameters from a single current-voltage characteristic using teaching learning based optimization algorithm," Applied Energy, vol. 119, pp. 384-393, 2014.

[59] S. Li, W. Gong, X. Yan et al., "Parameter extraction of photovoltaic models using an improved teaching- learning-based optimization," Energy Conversion and Management, vol. 186, pp. 293-305, 2019.

[60] O. S. Elazab, H. M. Hasanien, M. A. Elgendy, and A. M. Abdeen, "Parameters estimation of single- and multiple-diode photovoltaic model using whale optimisation algorithm," IET Renewable Power Generation, vol. 12, no. 15, pp. 1755-1761, 2018.

[61] G. Xiong, J. Zhang, D. Shi, and Y. He, "Parameter extraction of solar photovoltaic models using an improved whale optimization algorithm," Energy Conversion and Management, vol. 174, pp. 388-405, 2018.

[62] K. Yu, J. Liang, B. Qu, Z. Cheng, and H. Wang, "Multiple learning backtracking search algorithm for estimating parameters of photovoltaic models," Applied Energy, vol. 226, pp. 408-422, 2018.

[63] Y. Zhang, Z. Jin, X. Zhao, and Q. Yang, "Backtracking search algorithm with Levy flight for estimating parameters of photovoltaic models," Energy Conversion and Management, vol. 208, p. $112615,2020$.

[64] K. M. el-Naggar, M. R. AlRashidi, M. F. AlHajri, and A. K. alOthman, "Simulated annealing algorithm for photovoltaic parameters identification," Solar Energy, vol. 86, no. 1, pp. 266-274, 2012.

[65] S. Li, W. Gong, and Q. Gu, "A comprehensive survey on metaheuristic algorithms for parameter extraction of photovoltaic models," Renewable and Sustainable Energy Reviews, vol. 141, p. 110828, 2021. 